\title{
Rehabilitation Following Hip Fracture
}

\author{
Suzanne M. Dyer, Monica R. Perracini, Toby Smith, \\ Nicola J. Fairhall, Ian D. Cameron, Catherine Sherrington, \\ and Maria Crotty
}

\subsection{The Need for Increased Provision of Rehabilitation Worldwide}

The World Health Organization has recently highlighted a substantial unmet need for rehabilitation worldwide, with a 2017 Call-to-Action to increase the role of rehabilitation in health care as an essential component of integrated health services [1]. It was acknowledged that there is a profound unmet need for rehabilitation,

This chapter is a component of Part 3: Pillar II.

For an explanation of the grouping of chapters in this book, please see Chapter 1: "The multidisciplinary approach to fragility fractures around the world—an overview".

\author{
S. M. Dyer · M. Crotty $(\bowtie)$ \\ Rehabilitation, Aged and Extended Care, College of Medicine and Public Health, Flinders \\ University, Adelaide, SA, Australia \\ e-mail: sue.dyer@flinders.edu.au; maria.crotty@ flinders.edu.au \\ M. R. Perracini \\ Universidade Cidade de São Paulo, São Paulo, Brazil \\ e-mail: monica.perracini@unicid.edu.br \\ T. Smith \\ Department of Orthopaedics, Rheumatology and Musculoskeletal Sciences, University of \\ Oxford, Oxford, UK \\ e-mail: toby.smith@ndorms.ox.ac.uk

\section{N. J. Fairhall · C. Sherrington} \\ Institute for Musculoskeletal Health, The University of Sydney and Sydney Local Health \\ District, Sydney, NSW, Australia \\ e-mail: nicola.fairhall@ sydney.edu.au; cathie.sherrington@sydney.edu.au

\section{D. Cameron} \\ John Walsh Centre for Rehabilitation Research, Kolling Institute, Northern Sydney Local \\ Health District, St Leonards, NSW, Australia \\ e-mail: ian.cameron@sydney.edu.au


particularly in LMICs. There was a call for greater access to rehabilitation services recognising it as an essential part of the health system rather than an optional extra. Rehabilitation is "an investment in human capital that contributes to health, economic and social development" and there is under-prioritisation by governments with an absence of planning for services at a national and subnational level. The call to action includes "greater awareness and advocacy, increased investment into rehabilitation workforce and infrastructure, and improved leadership and governance structures". This includes increasing networks and partnerships in rehabilitation, particularly between LMICs and high-income countries.

\subsection{The Principles of Rehabilitation Programmes after Hip Fracture}

After a hip fracture operation, an older person's recovery is enhanced if they are provided with an optimistic, well-coordinated rehabilitation programme. Recovery after hip fracture starts on admission when the patient and family receive realistic information on the likely course and time of discharge. The earlier patient goals and expectations can be explored and information on barriers or supports for recovery of independence identified, the more likely it is that an individual will retain a sense of control and self-efficacy which is likely to be associated with better outcomes [2, $3]$. Consistent information on the planned rehabilitation programme is important as most people will have a recovery pathway which extends for several months across hospital and community settings [4].

During the acute hospital stay (ideally on an orthogeriatrics ward), along with secondary prevention treatments for osteoporosis, a rehabilitation pathway should be established and outlined to the patient and family. Rehabilitation involves diagnosing and treating impairments, preventing and treating complications, slowing loss of function and where this is not possible, compensating for lost functions (e.g. prescribing walking aids, bathroom adaptations, additional home help) [5]. Several systematic reviews and meta-analyses have demonstrated that rehabilitation programmes improve outcomes for patients after hip fracture compared to simply letting time take its course [6-8]. However, the components of recovery/rehabilitation programmes vary, including the length of time and the settings where programmes are delivered (home, inpatient units, outpatients). Standard management of hip fracture patients also varies between different countries. An audit in the UK reported that $70 \%$ of hip fracture patients receive orthogeriatrician assessment and $92 \%$ a falls assessment. These figures were only $27 \%$ for orthogeriatrician assessment and $4 \%$ for falls assessment in a tertiary hospital in Beijing [9].

In clinical practice, the cornerstone of a rehabilitation approach is a team of various disciplines (physiotherapy, occupational therapy, nutrition, social work, psychology, medicine) who meet regularly, set goals, review progress towards these goals with the patient and assess outcomes. The chance of recovery is maximised if the following elements are incorporated into the clinical approach:

- Assessment: identification of problems to be addressed, which involves understanding the premorbid level of functioning and understanding the current comorbidities (e.g. delirium). 
- Goal-setting: identifying what can be improved and what cannot. In particular, assessing what level of mobility and independence in bathing and dressing is likely to be achieved in the short, medium and long term. Similarly, identifying what informal and formal supports are available to help recovery.

- Treatment: intervening to improve medical and functional problems (such as pain, vitamin D deficiency, undernutrition, depression) as well as physical and psychosocial interventions to meet the rehabilitation goals.

- Evaluation: reviewing the effectiveness of interventions (i.e. reassessment).

- Planning: organising support services; providing self-management strategies for patients and carers.

The World Health Organization (WHO) International Classification of Functioning, Disability and Health (ICF) framework provides a standardised framework for the classification and description of health, functioning and disability [10]. It moves away from the idea that disability is simply the consequence of disease or ageing towards an approach that acknowledges factors created by the social environment and it attempts to explicitly identify barriers and facilitators to social inclusion. Functioning and disability are seen as multidimensional concepts, relating to:

- Body functions (physiological and psychological functions of body systems) and structures (anatomical parts of the body such as organs, limbs and their components);

- Activities people do and the life areas in which they participate;

- Factors in people's environment (physical, social and attitudinal) which can be barriers or facilitators to functioning.

If this approach is applied to a person who suffers a hip fracture, their disability will be assessed and ranked according to the ICF framework components of health domains (e.g. seeing, hearing, walking, memory) and health-related domains (e.g. their ability to access transport, their level of education and social interactions). Figure 12.1 shows an individual's functioning or disability as a dynamic interaction between health conditions and contextual factors, which encompass both environmental and personal factors [10].

\subsection{What Is Known about the Pattern of Recovery Following Hip Fracture?}

Talking to people with hip fractures and their families and providing realistic information on approximate expected recovery trajectories allows them to plan. However, it is complex for clinicians to apply evidence from cohort studies to individual patients as the cohorts are heterogeneous and patients have received varying amounts and types of rehabilitation.

Cohort studies suggest that following hip fracture, only $40-60 \%$ of people who survive are likely to recover their pre-fracture level of mobility [11]. Up to $70 \%$ may recover their level of independence for basic activities of daily living, but this is variable and less than half of all people experiencing hip fracture may regain their ability to perform instrumental ADLs. In Western nations, approximately 10-20\% of patients move to a residential care facility following hip fracture. The extent to which these outcomes can be improved with greater access to rehabilitation is not clear. 


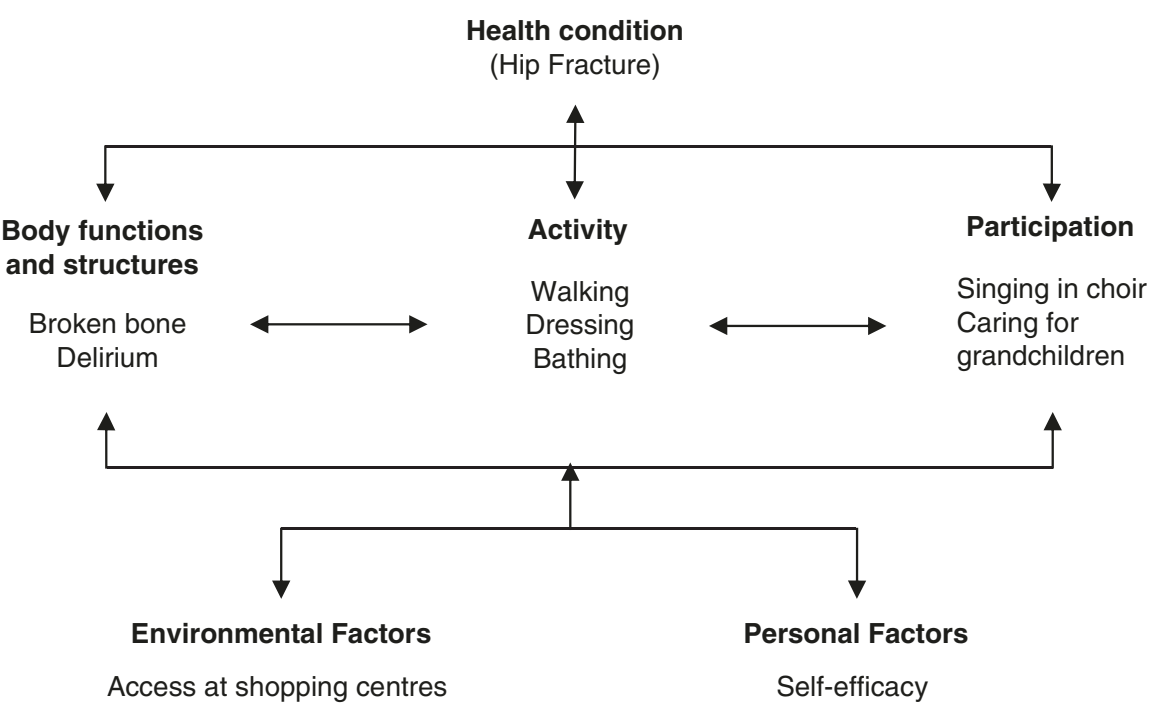

Fig. 12.1 Interactions between the components of the World Health Organization (WHO) International Classification of Functioning, Disability and Health (ICF) framework. Source: WHO $2001[10]$

Magaziner et al. described the sequence of recovery across eight different functional abilities following hip fracture [12]. Upper extremity activities of daily living, depression and cognitive function reached maximum recovery within 4 months. Most recovery of gait and balance occurred in the first 6 months, with maximum recovery occurring by 9 months; recovery of instrumental ADLs (such as shopping, preparing meals, house cleaning and handling money) took up to a year. It also took approximately a year for recovery of lower limb function and just over 14 months for walking $3 \mathrm{~m}$ without assistance. It seems that the majority of patients who recover their pre-fracture walking and basic activities of daily living do so within the first 6 months after fracture [13], but the role of long-term therapy in recovery pathways is yet to be well investigated.

In LMICs outcomes may be poorer due to reduced rates of optimal management, particularly due to reduced access to rehabilitation services [1]. In many LMICs standard hip fracture management pathways are not established [14]. Barriers to providing rehabilitation services in LMICs are discussed in more detail in Tables 12.10 and 12.11. An audit of practice in China (considered an upper MIC) demonstrated reduced access to surgery and orthogeriatric services at a Beijing hospital in comparison to the United Kingdom [9]. Since that time a retrospective before-after study has demonstrated that improvements in both time to surgery and orthogeriatric management are possible with implementation of a comanagement care plan [15]. 


\subsection{Factors Associated with Poor Outcomes After Hip Fracture}

Some types of patients with hip fracture appear to be at particular risk of poor outcomes - these include male patients, people living in supported accommodation, those with poorer mobility pre-fracture and those with depression or dementia [13, $16,17]$. People with dementia are also less likely to receive rehabilitation $[17,18]$. For people with dementia who receive rehabilitation, improvements comparable to other populations are achievable but this may take longer [19]. Although mortality following hip fracture has been found to be higher in men than women, recovery of mobility is unaffected by gender $[17,20]$.

Delirium is very common after hip fracture and although it is associated with poorer outcomes, routine assessment by rehabilitation staff remains uncommon [13]. In one prospective study, delirium remained in $39 \%$ of people with hip fracture at discharge from hospital and in 32\% 1 month after fracture [21]. Even after controlling for pre-fracture physical and cognitive frailty those people who had delirium were twice as likely to have poor functional outcomes (in terms of mobility and recovery of activities of daily living) than those without [21].

Those who are older are more likely to have poorer mobility, need assistance at home, lose their ability to go outside on their own, cook their own dinner and be unable to prepare their own breakfast $[17,20]$. Low food intake post-operatively, poor nutrition and malnourishment pre-operatively are associated with worse recovery of mobility and function [22, 23]. A systematic review of nutritional interventions found low-quality evidence to support the effectiveness of multi-nutrient supplements started before or soon after surgery in older people recovering from hip fracture to prevent complications [24]. Amongst nursing home residents, the factors most strongly associated with death or new total mobility dependence are being aged more than 90, having very severe cognitive impairment and receiving nonoperative management of the hip fracture [25]. Longer lengths of stay, re-hospitalisation, older age, chronic or acute cognitive deficits and depressive symptoms while in hospital are also predictive of poorer recovery of mobility and activities of daily living [13].

\subsection{Key Elements of a Rehabilitation Pathway}

After the immediate post-operative period, a rehabilitation pathway should be followed that includes the elements addressed in Table 12.1. In particular, there is a need to assess frailty, establish goals to maximise mobility and other aspects of function, assess the requirement of aids (using occupational therapy services where available) and determine strategies to support and improve independence in activities of daily living [26]. Medication management should ensure all prescribed medications are necessary, minimise the use of antipsychotics and sedatives and ensure adequate pain management. Osteoporosis should be treated as appropriate and falls prevention strategies reinforced with both patients and families. 
Table 12.1 Key elements of a typical rehabilitation pathway, based on the Alberta Hip Fracture Restorative Care Pathway [26]

\begin{tabular}{|c|c|}
\hline & \\
\hline Frailty & $\begin{array}{l}\text { Undertake frailty assessment, instigate interventions as appropriate, involve } \\
\text { patient in establishing goals to maximise function and achieve safe discharge }\end{array}$ \\
\hline $\begin{array}{l}\text { Activities of } \\
\text { daily living }\end{array}$ & $\begin{array}{l}\text { Ensure progression in recovery of pre-fracture level of independence, aiming } \\
\text { for further improvement depending on tolerance } \\
\text { Assess need for aids and develop strategies to improve independence } \\
\text { Demonstrate safe transfer using aids and equipment as appropriate } \\
\text { Ensure there is adequate support in the home environment in terms of } \\
\text { assistance from a caregiver or service } \\
\text { Recommend the family consider a medical alert system if available and } \\
\text { appropriate } \\
\text { Bathing and grooming: Encourage and support independence, bathing and } \\
\text { grooming out of bed with assistance if necessary } \\
\text { Dressing: Support getting out of bed and dressed daily, using dressing aids } \\
\text { as necessary } \\
\text { Toileting: Encourage regular toileting to promote continence, toileting } \\
\text { should be in the bathroom, not using bedpans or urinals } \\
\text { Eating: A high protein/calorie diet should be continued and meals taken in a } \\
\text { chair or dining room. An oral nutritional supplement should be considered } \\
\text { Support for activities of daily living should be provided after discharge. } \\
\text { Appropriate home equipment should be provided (mobility aid, raised toilet } \\
\text { seat and toilet surround and other items as required) }\end{array}$ \\
\hline Mobility & $\begin{array}{l}\text { Consider conducting an assessment of mobility/activities of daily living to } \\
\text { enable monitoring of recovery of mobility (e.g. the Timed Up and Go test, } \\
\text { Barthel Index of Activities of Daily Living) } \\
\text { Exercise incorporating strengthening, balance and functional components } \\
\text { should be continued after discharge } \\
\text { Walking with or without an aid for at least } 50-100 \mathrm{~m} \text { should be undertaken } \\
\text { at least three times daily, or as appropriate depending on pre-fracture } \\
\text { mobility } \\
\text { Capacity to walk the distance required to attend meals in the home setting } \\
\text { should be demonstrated } \\
\text { Ensure ability to manage stairs if necessary and to mobilise safely outside } \\
\text { the home in all weather conditions, uneven surfaces, curbs, etc. } \\
\text { Arrange further mobility training after hospital discharge }\end{array}$ \\
\hline Medications & $\begin{array}{l}\text { A review of all medications should have been undertaken on admission, } \\
\text { polypharmacy should be addressed } \\
\text { Use of sedatives and antipsychotics should be minimised or ceased and } \\
\text { doses should be regularly reviewed } \\
\text { Medication should be adequate for pain control to enable optimal } \\
\text { independence in activities of daily living }\end{array}$ \\
\hline $\begin{array}{l}\text { Cognitive ar } \\
\text { mental statu }\end{array}$ & $\begin{array}{l}\text { Strategies to prevent and treat delirium should be continued, including } \\
\text { ensuring appropriate use of vision and hearing aids, fluid enhancement, } \\
\text { orientation, optimising mobility and non-pharmacological sleep supporting } \\
\text { strategies. Behaviour monitoring should be undertaken if necessary } \\
\text { Activity should be encouraged for those with dementia or depression, in } \\
\text { terms of ambulation, exercise and social participation } \\
\text { Caregivers should be provided with support and access to community } \\
\text { resources as appropriate }\end{array}$ \\
\hline $\begin{array}{l}\text { Prevention of } \\
\text { further falls/ } \\
\text { fractures }\end{array}$ & $\begin{array}{l}\text { Osteoporosis management should be considered if this hasn't already } \\
\text { occurred and continued post-discharge } \\
\text { Fall prevention strategies should be instigated and the use of hip protectors } \\
\text { (if available) considered }\end{array}$ \\
\hline
\end{tabular}




\subsection{What Programmes Should We Recommend to Help with Recovery?}

It is widely recognised that a vicious cycle can occur after a hip fracture where pain and hospitalisation result in disuse atrophy of muscles and general deconditioning which increases the risk of immobility and new falls and fractures [27]. While national clinical guidelines recommend providing balance and strengthening exercise $[28,29]$, it is often unclear how much should be provided, what components of a rehabilitation programme are crucial and how long this programme should be provided for. Analysis of the components of interventions found to be effective in randomised trials can assist in addressing this uncertainty.

The characteristics of all randomised controlled trials of multidisciplinary rehabilitation approaches reporting impact on patient-centred outcomes or mortality (Table 12.2) and exercise and mobility training programmes (Table 12.3), within different settings as identified by systematic review to 2019, are presented below $[30,31]$. The quality of the trials is represented with the Physiotherapy Evidence Database (PEDro) score (see https://www.pedro.org.au/), which scores ten items reflecting trial design including randomisation, blinding, balance in baseline characteristics and follow-up. Details of the components of the programmes that have been demonstrated to be effective at improving mobility or function in randomised controlled trials are shown in Table 12.4 (multidisciplinary interventions in hospital or hospital and community setting), Table 12.5 (exercise programmes conducted in hospital settings), Table 12.6 (community-based multidisciplinary interventions) and Table 12.7 (community-based exercise programmes).

\subsubsection{In-hospital Rehabilitation}

Multidisciplinary programmes have been researched over a long period of time with significant changes to the components of treatment programs. Some trials provide a comparison to a usual care control group while others have a standard rehabilitation programme as the control group. Furthermore, some multidisciplinary programs begin soon after admission (e.g. Prestmo et al. [40]) and others include both in hospital rehabilitation and community components (e.g. Cameron et al. [45]). A 1988 trial demonstrated improvements in function on discharge for provision of multidisciplinary care in comparison to standard orthopaedic care [37].

Table 12.4 shows the characteristics of hospital-based and hospital-plus community-based multidisciplinary rehabilitation interventions in studies with demonstrated effectiveness. Four trials of programmes delivered solely in a hospital setting have demonstrated effectiveness on patient-centred outcomes overall (Table 12.4). One effective in-hospital programme with comprehensive, multidisciplinary geriatric care including early mobilisation, and daily training and a follow-up assessment at 4 months, demonstrated improvements in function at 1 year [42]. Another trial demonstrated improvements in mobility with orthogeriatric care for a subgroup who were living at home [44]. A third trial demonstrated reduced rates of delirium with daily geriatrician visits [38]. 
Table 12.2 Characteristics of trials of multidisciplinary rehabilitation approaches reporting impact on patient-centred outcomes or mortality

\begin{tabular}{|c|c|c|c|c|c|c|}
\hline Study & Setting & $\begin{array}{l}\text { Sample } \\
\text { size }\end{array}$ & PEDro & $\begin{array}{l}\text { Patient-centred } \\
\text { outcomes }\end{array}$ & $\begin{array}{l}\text { Characteristics of } \\
\text { intervention }\end{array}$ & Comparator \\
\hline \multicolumn{7}{|c|}{ Hospital-based programmes } \\
\hline $\begin{array}{l}\text { Baroni } 2019 \\
\text { [32] }\end{array}$ & $\mathrm{H}$ & 430 & $6^{a}$ & Mortality & $\begin{array}{l}\text { Geriatric } \\
\text { consultation } \\
\text { service }\end{array}$ & $\begin{array}{l}\text { Orthogeriatric } \\
\text { comanagement }\end{array}$ \\
\hline $\begin{array}{l}\text { Chong } 2013 \\
\text { [33] }\end{array}$ & $\mathrm{H}$ & 162 & $5^{a}$ & $\begin{array}{l}\text { Function, mobility, } \\
\text { institutionalisation, } \\
\text { mortality, quality } \\
\text { of life }\end{array}$ & $\begin{array}{l}\text { Integrated care } \\
\text { pathway: Usual } \\
\text { care plus } \\
\text { structured therapy } \\
\text { assessments and } \\
\text { checklists }\end{array}$ & $\begin{array}{l}\text { Usual care within } \\
\text { multi D team }\end{array}$ \\
\hline $\begin{array}{l}\text { Fordham } \\
1986[34]\end{array}$ & $\mathrm{H}$ & 108 & $5^{\mathrm{a}}$ & $\begin{array}{l}\text { Poor outcome at } \\
\text { discharge, } \\
\text { mortality, function, } \\
\text { mobility }\end{array}$ & $\begin{array}{l}\text { Joint geriatric and } \\
\text { orthopaedic } \\
\text { management }\end{array}$ & $\begin{array}{l}\text { Orthopaedic } \\
\text { management }\end{array}$ \\
\hline $\begin{array}{l}\text { Galvard } \\
1995[35]\end{array}$ & $\mathrm{H}$ & 378 & 4 & $\begin{array}{l}\text { Poor outcome at } \\
\text { discharge, }{ }^{b} \\
\text { mortality }\end{array}$ & $\begin{array}{l}\text { Geriatric rehab } \\
\text { within geriatric } \\
\text { hospital }\end{array}$ & $\begin{array}{l}\text { Usual } \\
\text { orthopaedic care }\end{array}$ \\
\hline $\begin{array}{l}\text { Gilchrist } \\
1988[36]\end{array}$ & $\mathrm{H}$ & 222 & 5 & $\begin{array}{l}\text { Poor outcome at } \\
\text { discharge, } \\
\text { mortality }\end{array}$ & $\begin{array}{l}\text { Combined } \\
\text { geriatric- } \\
\text { orthopaedic care } \\
\text { in special } \\
\text { designated unit }\end{array}$ & $\begin{array}{l}\text { Usual } \\
\text { orthopaedic care } \\
\text { in orthopaedic } \\
\text { ward }\end{array}$ \\
\hline $\begin{array}{l}\text { Kennie } 1988 \\
{[37]}\end{array}$ & $\mathrm{H}$ & 108 & 6 & $\begin{array}{l}\text { Poor outcome at } \\
\text { discharge and poor } \\
\text { outcome at } \\
\text { long-term } \\
\text { follow-up, }{ }^{\text {c }} \\
\text { mortality, function }\end{array}$ & $\begin{array}{l}\text { Multi D care in } \\
\text { orthopaedic beds } \\
\text { at peripheral } \\
\text { hospital, plus } \\
\text { allied health visits }\end{array}$ & $\begin{array}{l}\text { Usual } \\
\text { orthopaedic care } \\
\text { in orthopaedic } \\
\text { ward, plus allied } \\
\text { health }\end{array}$ \\
\hline $\begin{array}{l}\text { Marcantonio } \\
2001[38]\end{array}$ & $\mathrm{H}$ & 126 & $8^{\mathrm{a}}$ & Delirium & $\begin{array}{l}\text { Proactive } \\
\text { geriatrics } \\
\text { consultation }\end{array}$ & Usual care \\
\hline $\begin{array}{l}\text { Naglie } 2002 \\
\text { [39] }\end{array}$ & $\mathrm{H}$ & 280 & 7 & $\begin{array}{l}\text { Poor outcome at } \\
\text { long-term } \\
\text { follow-up, } \\
\text { mortality, function, } \\
\text { mobility }\end{array}$ & $\begin{array}{l}\text { Multi D care: } \\
\text { Routine } \\
\text { post-operative } \\
\text { surgical care, } \\
\text { daily geriatrician } \\
\text { care, allied health, } \\
\text { emphasis on } \\
\text { prevention, } \\
\text { mobilisation, } \\
\text { self-care, } \\
\text { discharge } \\
\text { planning }\end{array}$ & $\begin{array}{l}\text { Usual care on } \\
\text { orthopaedic unit }\end{array}$ \\
\hline $\begin{array}{l}\text { Prestmo } \\
2015[40]\end{array}$ & $\mathrm{H}$ & 397 & 6 & $\begin{array}{l}\text { Mobility, quality of } \\
\text { life, function }\end{array}$ & $\begin{array}{l}\text { Comprehensive } \\
\text { geriatric care }\end{array}$ & $\begin{array}{l}\text { Usual } \\
\text { orthopaedic care }\end{array}$ \\
\hline $\begin{array}{l}\text { Sanchez } \\
\text { Ferrin } 1999 \\
{[41]}\end{array}$ & $\mathrm{H}$ & 206 & $6^{\mathrm{a}}$ & $\begin{array}{l}\text { Function, mobility, } \\
\text { mortality, } \\
\text { institutionalisation }\end{array}$ & $\begin{array}{l}\text { Evaluated by the } \\
\text { functional } \\
\text { geriatric unit }\end{array}$ & Usual care \\
\hline
\end{tabular}


Table 12.2 (continued)

\begin{tabular}{|c|c|c|c|c|c|c|}
\hline Study & Setting & $\begin{array}{l}\text { Sample } \\
\text { size }\end{array}$ & PEDro & $\begin{array}{l}\text { Patient-centred } \\
\text { outcomes }\end{array}$ & $\begin{array}{l}\text { Characteristics of } \\
\text { intervention }\end{array}$ & Comparator \\
\hline $\begin{array}{l}\text { Stenvall } \\
2007[42]\end{array}$ & $\mathrm{H}$ & 199 & 6 & $\begin{array}{l}\text { Poor outcome at } \\
\text { discharge and } \\
\text { long-term } \\
\text { follow-up, } \\
\text { mortality, function, } \\
\text { mobility, } \\
\text { independent living }\end{array}$ & $\begin{array}{l}\text { Comprehensive } \\
\text { geriatric care with } \\
\text { assessment at } \\
\text { 4-months }\end{array}$ & $\begin{array}{l}\text { Usual care on } \\
\text { specialist } \\
\text { orthopaedic ward } \\
\text { No 4-month } \\
\text { assessment }\end{array}$ \\
\hline $\begin{array}{l}\text { Uy } 2008 \\
{[43]}\end{array}$ & $\mathrm{H}$ & 10 & 6 & Function, mobility & $\begin{array}{l}\text { Inpatient multi D } \\
\text { rehab programme, } \\
\text { using system of } \\
\text { accelerated rehab }\end{array}$ & $\begin{array}{l}\text { Usual care } \\
\text { (discharge back } \\
\text { to NH soon after } \\
\text { surgery) }\end{array}$ \\
\hline $\begin{array}{l}\text { Watne } 2014 \\
{[44]}\end{array}$ & $\mathrm{H}$ & 329 & $7^{\mathrm{a}}$ & $\begin{array}{l}\text { Delirium, } \\
\text { mortality, mobility }\end{array}$ & $\begin{array}{l}\text { Patients treated in } \\
\text { acute geriatric } \\
\text { ward: } \\
\text { Comprehensive } \\
\text { geriatric } \\
\text { assessment, daily } \\
\text { multi D meetings }\end{array}$ & $\begin{array}{l}\text { Usual care in } \\
\text { orthopaedic ward }\end{array}$ \\
\hline \multicolumn{7}{|c|}{ Hospital- and community-based programmes } \\
\hline $\begin{array}{l}\text { Cameron } \\
1993[45]\end{array}$ & $\mathrm{H} \& \mathrm{C}$ & 252 & 6 & $\begin{array}{l}\text { Poor outcome at } \\
\text { discharge and } \\
\text { long-term } \\
\text { follow-up, }{ }^{\mathrm{f}} \\
\text { mortality, function }\end{array}$ & $\begin{array}{l}\text { Accelerated rehab } \\
\text { and early } \\
\text { discharge }\end{array}$ & Usual care \\
\hline $\begin{array}{l}\text { Crotty } 2003 \\
{[46]}\end{array}$ & $\mathrm{H} \& \mathrm{C}$ & 66 & 6 & $\begin{array}{l}\text { Poor outcome at } \\
\text { long-term } \\
\text { follow-up, } \\
\text { mortality, quality } \\
\text { of life, function, } \\
\text { mobility }\end{array}$ & $\begin{array}{l}\text { Ambulatory } \\
\text { geriatric } \\
\text { interdisciplinary } \\
\text { rehab programme }\end{array}$ & Usual care \\
\hline $\begin{array}{l}\text { Huusko } \\
2002[47]\end{array}$ & $\mathrm{H} \& \mathrm{C}$ & 260 & 5 & $\begin{array}{l}\text { Poor outcome at } \\
\text { long-term } \\
\text { follow-up, }{ }^{\mathrm{f}} \\
\text { mortality, function }\end{array}$ & $\begin{array}{l}\text { Intensive geriatric } \\
\text { rehab in hospital, } \\
\text { multi D geriatric } \\
\text { team, physio } \\
\text { sessions and } \\
\text { ongoing treatment } \\
\text { at home } \\
\text { post-discharge }\end{array}$ & $\begin{array}{l}\text { Discharge to } \\
\text { local community } \\
\text { hospitals, } \\
\text { treatment by } \\
\text { GPs, } \\
\text { physiotherapists }\end{array}$ \\
\hline $\begin{array}{l}\text { Jette } 1987 \\
{[48]}\end{array}$ & $\mathrm{H} \& \mathrm{C}$ & 68 & 2 & $\begin{array}{l}\text { Function (ADLs } \\
\text { and social } \\
\text { function) }\end{array}$ & $\begin{array}{l}\text { Intensive rehab } \\
\text { programme: } \\
\text { Standard } \\
\text { programme plus } \\
\text { individualised } \\
\text { patient and family } \\
\text { education, } \\
\text { comprehensive } \\
\text { assessment, } \\
\text { weekly team } \\
\text { meetings, home } \\
\text { visit and telephone } \\
\text { calls } \\
\text { post-discharge }\end{array}$ & $\begin{array}{l}\text { Standard } \\
\text { post-surgical } \\
\text { rehab } \\
\text { programme, } \\
\text { including } \\
\text { follow-up visits } \\
\text { to clinic at } \\
6 \text { weeks, } 3,6 \text {, } \\
\text { and } 12 \text { months } \\
\text { post-discharge }\end{array}$ \\
\hline
\end{tabular}


Table 12.2 (continued)

\begin{tabular}{|c|c|c|c|c|c|c|}
\hline Study & Setting & $\begin{array}{l}\text { Sample } \\
\text { size }\end{array}$ & PEDro & $\begin{array}{l}\text { Patient-centred } \\
\text { outcomes }\end{array}$ & $\begin{array}{l}\text { Characteristics of } \\
\text { intervention }\end{array}$ & Comparator \\
\hline $\begin{array}{l}\text { Karlson } \\
2016 \text { [49] }\end{array}$ & $\mathrm{H} \& \mathrm{C}$ & 205 & 8 & $\begin{array}{l}\text { Mobility, function, } \\
\text { delirium, quality of } \\
\text { life }\end{array}$ & $\begin{array}{l}\text { Usual care and } \\
\text { geriatric multi D } \\
\text { home rehab, with } \\
\text { aim of early } \\
\text { discharge, } \\
\text { individually } \\
\text { designed, } \\
\text { conducted by } \\
\text { multi D team, for } \\
10 \text { weeks }\end{array}$ & $\begin{array}{l}\text { Usual care and } \\
\text { rehab in geriatric } \\
\text { ward with } \\
\text { comprehensive } \\
\text { geriatric } \\
\text { assessment, } \\
\text { post-discharge } \\
\text { primary HC and } \\
\text { outpatient rehab } \\
\text { at } 3 \text { months as } \\
\text { needed }\end{array}$ \\
\hline $\begin{array}{l}\text { Shyu } 2010 \\
{[50]}\end{array}$ & $\mathrm{H} \& \mathrm{C}$ & 162 & 7 & $\begin{array}{l}\text { Poor outcome at } \\
\text { follow-up, }{ }^{\mathrm{g}} \\
\text { function, mortality, } \\
\text { mobility, quality of } \\
\text { life }\end{array}$ & $\begin{array}{l}\text { Interdisciplinary } \\
\text { programme }\end{array}$ & $\begin{array}{l}\text { Usual care on } \\
\text { trauma or } \\
\text { orthopaedic } \\
\text { ward. No } \\
\text { follow-up care } \\
\text { post-discharge }\end{array}$ \\
\hline $\begin{array}{l}\text { Shyu } 2013 \\
{[51]}\end{array}$ & $\mathrm{H} \& \mathrm{C}$ & 299 & $6^{\mathrm{a}}$ & Function & $\begin{array}{l}1 . \\
\text { Interdisciplinary } \\
\text { care } \\
\text { 2. Comprehensive } \\
\text { care }\end{array}$ & $\begin{array}{l}\text { Usual care: } \\
\text { Current routine } \\
\text { care of hip } \\
\text { fractured elders } \\
\text { in Taiwan, no } \\
\text { continuation of } \\
\text { rehab in home } \\
\text { setting }\end{array}$ \\
\hline $\begin{array}{l}\text { Singh } 2012 \\
\text { [52] }\end{array}$ & $\mathrm{H} \& \mathrm{C}$ & 124 & 5 & $\begin{array}{l}\text { Function, mortality, } \\
\text { residence, mobility, } \\
\text { quality of life }\end{array}$ & $\begin{array}{l}\text { Geriatrician } \\
\text { supervised, } \\
\text { high-intensity } \\
\text { resistance exercise } \\
\text { and targeted multi } \\
\text { D interventions }\end{array}$ & $\begin{array}{l}\text { Standard care: } \\
\text { Orthogeriatric } \\
\text { care, rehab } \\
\text { service and } \\
\text { physio }\end{array}$ \\
\hline $\begin{array}{l}\text { Swanson } \\
1998 \text { [53] }\end{array}$ & $\mathrm{H} \& \mathrm{C}$ & 71 & 6 & $\begin{array}{l}\text { Poor outcome at } \\
\text { discharge, } \\
\text { mortality, mobility, } \\
\text { function }\end{array}$ & $\begin{array}{l}\text { Accelerated rehab } \\
\text { programme } \\
\text { involving multi D } \\
\text { team }\end{array}$ & $\begin{array}{l}\text { Standard } \\
\text { orthopaedic } \\
\text { management. } \\
\text { Home visits as } \\
\text { needed } \\
\text { post-discharge }\end{array}$ \\
\hline $\begin{array}{l}\text { Vidan } 2005 \\
\text { [54] }\end{array}$ & $\mathrm{H} \& \mathrm{C}$ & 321 & 6 & $\begin{array}{l}\text { Poor outcome at } \\
\text { long-term } \\
\text { follow-up, }{ }^{\mathrm{c}} \\
\text { mortality, function, } \\
\text { mobility }\end{array}$ & $\begin{array}{l}\text { Multi D } \\
\text { care-Geriatric } \\
\text { team, assessments, } \\
\text { rehab specialist, } \\
\text { social worker, } \\
\text { comprehensive } \\
\text { treatment plan }\end{array}$ & $\begin{array}{l}\text { Usual } \\
\text { orthopaedic care. } \\
\text { Specialist } \\
\text { counselling as } \\
\text { required }\end{array}$ \\
\hline $\begin{array}{l}\text { Ziden } 2008 \\
\text { [55] }\end{array}$ & $\mathrm{H} \& \mathrm{C}$ & 102 & 6 & $\begin{array}{l}\text { Function, mobility, } \\
\text { quality of life }\end{array}$ & $\begin{array}{l}\text { Home Rehab } \\
\text { (HR) programme }\end{array}$ & $\begin{array}{l}\text { Conventional } \\
\text { Care }(\mathrm{CC}) \text { and } \\
\text { rehab, discharged } \\
\text { home or to } \\
\text { short-term NH }\end{array}$ \\
\hline \multicolumn{7}{|c|}{ Community-based programmes } \\
\hline $\begin{array}{l}\text { Crotty } 2019 \\
\text { [56] }\end{array}$ & $\mathrm{C}$ & 240 & $7^{\mathrm{a}}$ & $\begin{array}{l}\text { Mobility, quality of } \\
\text { life, function, } \\
\text { delirium }\end{array}$ & $\begin{array}{l}\text { Multi D post-op } \\
\text { rehab programme } \\
\text { within NH }\end{array}$ & $\begin{array}{l}\text { Usual care within } \\
\mathrm{NH}\end{array}$ \\
\hline
\end{tabular}


Table 12.2 (continued)

\begin{tabular}{|c|c|c|c|c|c|c|}
\hline Study & Setting & $\begin{array}{l}\text { Sample } \\
\text { size }\end{array}$ & PEDro & $\begin{array}{l}\text { Patient-centred } \\
\text { outcomes }\end{array}$ & $\begin{array}{l}\text { Characteristics of } \\
\text { intervention }\end{array}$ & Comparator \\
\hline $\begin{array}{l}\text { Ryan } 2006 \\
\text { [57] }\end{array}$ & $\mathrm{C}$ & 71 & 6 & $\begin{array}{l}\text { Poor outcome at } \\
\text { long-term } \\
\text { follow-up, } \\
\text { mortality, function }\end{array}$ & $\begin{array}{l}\text { Intensive } \\
\text { treatment: } \geq 6 \\
\text { face-to-face } \\
\text { contacts pw from } \\
\text { members of a } \\
\text { multi D rehab } \\
\text { team }\end{array}$ & $\begin{array}{l}\text { Less intensive } \\
\text { treatment: } \leq 3 \\
\text { face-to-face } \\
\text { contacts pw with } \\
\text { members of } \\
\text { multi D rehab } \\
\text { team }\end{array}$ \\
\hline
\end{tabular}

Note: as identified by systematic review to October 2019. a Scored in duplicate by authors $A D L s$ activities of daily living, $C$ community only, $G P$ general practitioner, $H$ hospital only, $H \& C$ hospital and community, $H C$ health care, IADLs instrumental ADLs, multi D multidisciplinary, NA not available, $\mathrm{NH}$ nursing home, physio physiotherapist/physiotherapy, post-op post-operative, $p w$ per week, rehab rehabilitation

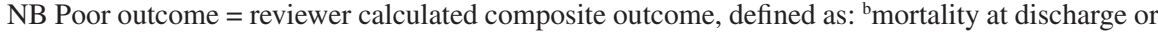
discharge to more dependent residence/ $\mathrm{NH}$, 'mortality at 12 months or living in more dependent residence/ $\mathrm{NH},{ }^{d}$ mortality at discharge, discharge to more dependent residence and decline in mobility, ${ }^{\mathrm{e}}$ mortality at discharge, not discharged to previous residence, not in same residence at 12 months and reduced ADLs, ${ }^{\mathrm{f}}$ mortality at discharge or discharge to institutional care, mortality at 12 months or institutional care, ${ }^{\mathrm{g}} 12$-month mortality, admission to institutional care or decline in function

Table 12.3 Characteristics of trials of exercise and mobility training programmes reporting impact on mobility or function outcomes ${ }^{\mathrm{a}}$

\begin{tabular}{|c|c|c|c|c|c|c|}
\hline Study & Setting & $\begin{array}{l}\text { Sample } \\
\text { size }\end{array}$ & PEDro & $\begin{array}{l}\text { Main mobility } \\
\text { outcome }\end{array}$ & $\begin{array}{l}\text { Characteristics of } \\
\text { intervention }\end{array}$ & Comparator \\
\hline \multicolumn{7}{|c|}{ Hospital-based programmes } \\
\hline $\begin{array}{l}\text { Kimmel } \\
2016[58]\end{array}$ & $\mathrm{H}$ & 92 & 7 & $\begin{array}{l}\text { Modified Iowa } \\
\text { Level of } \\
\text { Assistance } \\
\text { (mILOA) }\end{array}$ & $\begin{array}{l}\text { High-intensity } \\
\text { functional training }\end{array}$ & Usual care \\
\hline $\begin{array}{l}\text { Kronborg } \\
2017 \text { [59] }\end{array}$ & $\mathrm{H}$ & 90 & 7 & $\begin{array}{l}\text { Timed Up and } \\
\text { Go }\end{array}$ & $\begin{array}{l}\text { Progressive } \\
\text { resistance }\end{array}$ & Usual care \\
\hline $\begin{array}{l}\text { Mitchell } \\
2001[30]\end{array}$ & $\mathrm{H}$ & 80 & 5 & $\begin{array}{l}\text { Elderly Mobility } \\
\text { Scale }\end{array}$ & $\begin{array}{l}\text { High-intensity } \\
\text { progressive } \\
\text { resistance }\end{array}$ & Usual care \\
\hline $\begin{array}{l}\text { Monticone } \\
2018[31]\end{array}$ & $\mathrm{H}$ & 52 & 7 & $\begin{array}{l}\text { Western Ontario } \\
\text { and McMaster } \\
\text { Universities } \\
\text { Osteoarthritis } \\
\text { Index (WOMAC) }\end{array}$ & Balance exercises & $\begin{array}{l}\text { Usual care/ } \\
\text { open kinetic } \\
\text { chain exercises } \\
\text { in the supine } \\
\text { position }\end{array}$ \\
\hline $\begin{array}{l}\text { Moseley } \\
2009[60]\end{array}$ & $\mathrm{H}$ & 160 & 8 & PPME & $\begin{array}{l}\text { High-intensity } \\
\text { weight-bearing }\end{array}$ & Usual care \\
\hline $\begin{array}{l}\text { Ohoka } 2015 \\
{[61]}\end{array}$ & $\mathrm{H}$ & 27 & 4 & Gait speed & $\begin{array}{l}\text { Body weight- } \\
\text { supported treadmill } \\
\text { training }\end{array}$ & Usual care \\
\hline $\begin{array}{l}\text { Resnick } \\
2007 \text { [62] }\end{array}$ & $\mathrm{H}$ & 208 & 6 & $\begin{array}{l}\text { Self-efficacy } \\
\text { WES }\end{array}$ & $\begin{array}{l}\text { Exercise plus or } \\
\text { exercise only }\end{array}$ & Usual care \\
\hline $\begin{array}{l}\text { Sherrington } \\
2003 \text { [63] }\end{array}$ & $\mathrm{H}$ & 80 & 7 & PPME & Weight-bearing & $\begin{array}{l}\text { Non } \\
\text { weight-bearing }\end{array}$ \\
\hline $\begin{array}{l}\text { Van Ooijen } \\
2016 \text { [64] }\end{array}$ & $\mathrm{H}$ & 70 & 5 & $\begin{array}{l}\text { Elderly Mobility } \\
\text { Scale }\end{array}$ & $\begin{array}{l}\text { Treadmill vs. } \\
\text { adapted treadmill }\end{array}$ & Usual care \\
\hline
\end{tabular}


Table 12.3 (continued)

\begin{tabular}{|c|c|c|c|c|c|c|}
\hline Study & Setting & $\begin{array}{l}\text { Sample } \\
\text { size }\end{array}$ & PEDro & $\begin{array}{l}\text { Main mobility } \\
\text { outcome }\end{array}$ & $\begin{array}{l}\text { Characteristics of } \\
\text { intervention }\end{array}$ & Comparator \\
\hline \multicolumn{7}{|c|}{ Community-based programmes } \\
\hline $\begin{array}{l}\text { Binder } 2004 \\
{[65]}\end{array}$ & $\mathrm{H} \& \mathrm{C}$ & 90 & 7 & Modified PPT & $\begin{array}{l}\text { High-intensity } \\
\text { progressive } \\
\text { resistance }\end{array}$ & $\begin{array}{l}\text { Low-intensity } \\
\text { non- } \\
\text { progressive }\end{array}$ \\
\hline $\begin{array}{l}\text { Hauer } 2002 \\
{[66]}\end{array}$ & $\mathrm{H} \& \mathrm{C}$ & 28 & 6 & Tinetti's POMA & $\begin{array}{l}\text { High-intensity } \\
\text { progressive } \\
\text { resistance }\end{array}$ & $\begin{array}{l}\text { Placebo motor } \\
\text { activity }\end{array}$ \\
\hline $\begin{array}{l}\text { Langford } \\
2015 \text { [67] }\end{array}$ & $\mathrm{C}$ & 30 & 7 & Gait speed & $\begin{array}{l}\text { Additional post- } \\
\text { discharge } \\
\text { physiotherapist } \\
\text { telephone support } \\
\text { and coaching }\end{array}$ & Usual care \\
\hline $\begin{array}{l}\text { Latham } \\
2014[68]\end{array}$ & $\mathrm{H} \& \mathrm{C}$ & 232 & 6 & SPPB & $\begin{array}{l}\text { Home-based } \\
\text { exercise }\end{array}$ & $\begin{array}{l}\text { Attention } \\
\text { control }\end{array}$ \\
\hline $\begin{array}{l}\text { Magaziner } \\
2019 \text { [69] }\end{array}$ & $\mathrm{C}$ & 210 & $8^{c}$ & $\begin{array}{l}6 \text { min walk } \\
\text { distance }\end{array}$ & $\begin{array}{l}\text { Aerobic, strength, } \\
\text { balance and } \\
\text { functional training }\end{array}$ & $\begin{array}{l}\text { TENS and } \\
\text { range-of- } \\
\text { motion } \\
\text { exercises }\end{array}$ \\
\hline $\begin{array}{l}\text { Mangione } \\
2005[70]\end{array}$ & $\mathrm{C}$ & 41 & 5 & $\begin{array}{l}6 \text { min walk } \\
\text { distance }\end{array}$ & $\begin{array}{l}\text { Resistance or } \\
\text { aerobic exercise }\end{array}$ & Education \\
\hline $\begin{array}{l}\text { Mangione } \\
2010 \text { [71] }\end{array}$ & $\mathrm{C}$ & 26 & 7 & $\begin{array}{l}6 \text { min walk } \\
\text { distance }\end{array}$ & $\begin{array}{l}\text { Home-based } \\
\text { resistance }\end{array}$ & $\begin{array}{l}\text { Attention } \\
\text { control }\end{array}$ \\
\hline $\begin{array}{l}\text { Orwig } 2011 \\
{[72]}\end{array}$ & $\mathrm{C}$ & 180 & 6 & $\begin{array}{l}\text { Study primary } \\
\text { outcome: bone } \\
\text { mineral density; } \\
\text { mobility: } 6 \text { min } \\
\text { walk test }\end{array}$ & $\begin{array}{l}\text { Progressive } \\
\text { resistance and } \\
\text { aerobic }\end{array}$ & Usual care \\
\hline $\begin{array}{l}\text { Salpakoski } \\
2014 \text { [73] }\end{array}$ & $\mathrm{C}$ & 81 & 8 & $\begin{array}{l}\text { Short physical } \\
\text { performance } \\
\text { battery }\end{array}$ & $\begin{array}{l}\text { Progressive } \\
\text { resistance, balance } \\
\text { and functional }\end{array}$ & Usual care \\
\hline $\begin{array}{l}\text { Sherrington } \\
1997[74]\end{array}$ & $\mathrm{C}$ & 42 & 5 & Gait velocity & Weight-bearing & Usual care \\
\hline $\begin{array}{l}\text { Sherrington } \\
2004[75]\end{array}$ & $\mathrm{C}$ & 120 & 7 & $6 \mathrm{~m}$ walk time & $\begin{array}{l}\text { Weight-bearing or } \\
\text { non-weight-bearing }\end{array}$ & $\begin{array}{l}\text { No } \\
\text { intervention }\end{array}$ \\
\hline $\begin{array}{l}\text { Stasi } 2019 \\
{[76]}\end{array}$ & $\mathrm{C}$ & 96 & 7 & $\begin{array}{l}\text { Timed Up and } \\
\text { Go }\end{array}$ & $\begin{array}{l}\text { Progressive } \\
\text { resistance }\end{array}$ & Usual care \\
\hline $\begin{array}{l}\text { Sylliaas } \\
2011[77]\end{array}$ & $\mathrm{C}$ & 150 & 8 & $\begin{array}{l}6 \text { min walk } \\
\text { distance }\end{array}$ & $\begin{array}{l}\text { Progressive } \\
\text { resistance }\end{array}$ & $\begin{array}{l}\text { No } \\
\text { intervention }\end{array}$ \\
\hline $\begin{array}{l}\text { Sylliaas } \\
2012[78]\end{array}$ & $\mathrm{C}$ & 95 & 8 & $\begin{array}{l}6 \text { min walk } \\
\text { distance }\end{array}$ & $\begin{array}{l}\text { Prolonged } \\
\text { resistance }\end{array}$ & $\begin{array}{l}\text { No } \\
\text { intervention }\end{array}$ \\
\hline $\begin{array}{l}\text { Tsauo } 2005 \\
\text { [79] }\end{array}$ & $\mathrm{C}$ & 54 & 4 & Walking speed & $\begin{array}{l}\text { Home-based } \\
\text { physiotherapy }\end{array}$ & $\begin{array}{l}\text { Bedside } \\
\text { exercise }\end{array}$ \\
\hline $\begin{array}{l}\text { Williams } \\
2016[80]\end{array}$ & $\mathrm{C}$ & 61 & 8 & $\begin{array}{l}\text { Timed Up and } \\
\text { Go }\end{array}$ & $\begin{array}{l}\text { Additional } \\
\text { physiotherapy }\end{array}$ & Usual care \\
\hline
\end{tabular}

$C$ community only, $H$ hospital only, $H \& C$ hospital and community, $N$ no, $P P T$ Physical Performance Test, POMA Performance Oriented Mobility Assessment, PPME Physical Performance Mobility Examination $S P P B$ Short Physical Performance Battery, TENS transcutaneous electrical nerve stimulation, WES Walking Exercise Scale $Y$ yes

${ }^{a}$ Based on systematic-review of MEDLINE, EMBASE, CINAHL, CENTRAL and PEDro database search records from inception to April 2019 for randomised controlled trials of exercise-based programmes aiming to improve mobility in older people post-hip fracture reporting data suitable for inclusion in meta-analysis, with a minim PEDro score of 5 or more

${ }^{b}$ Only two out of three comparison groups examined exercise interventions ${ }^{\mathrm{c}} \mathrm{S}$ cored in duplicate by current authors 


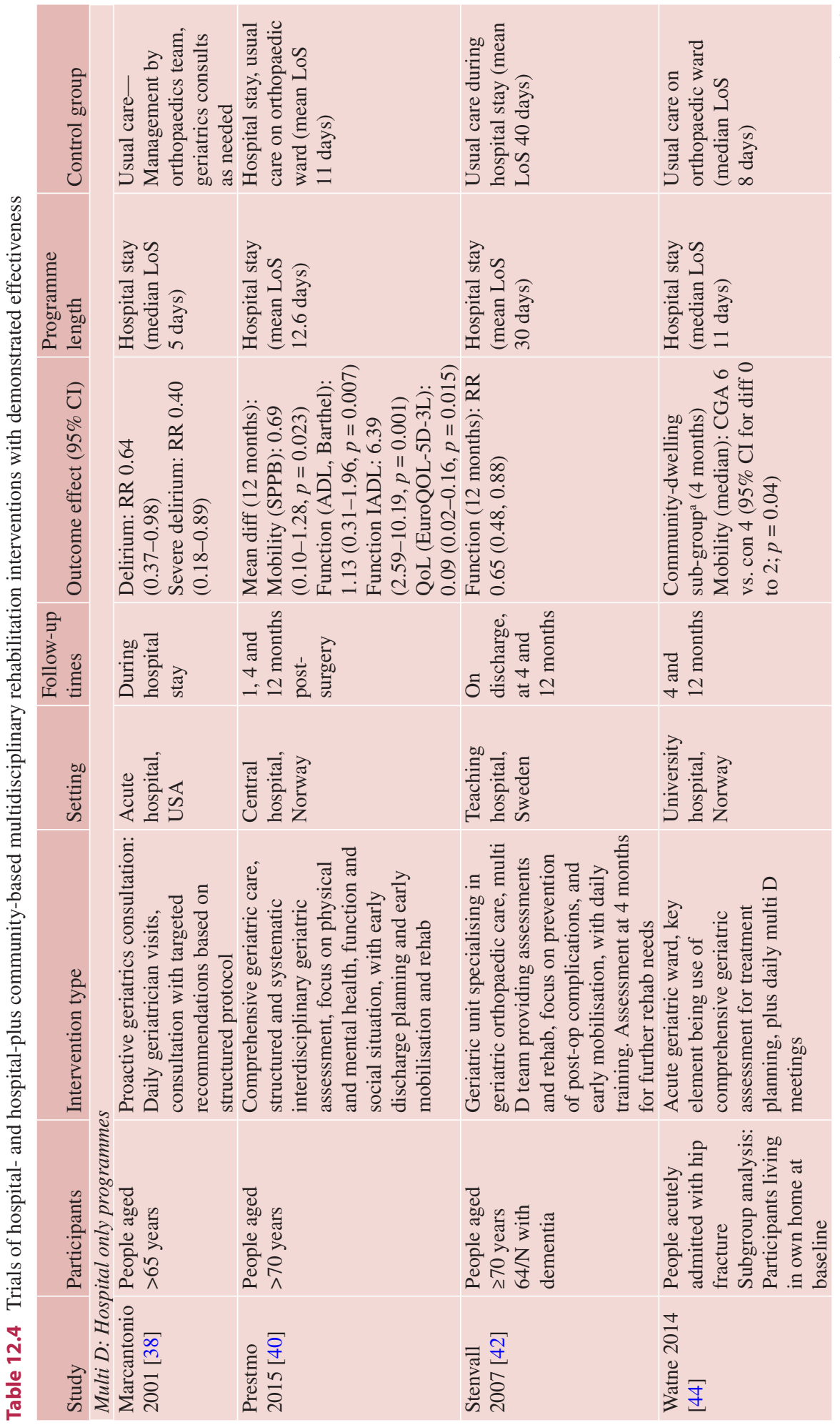




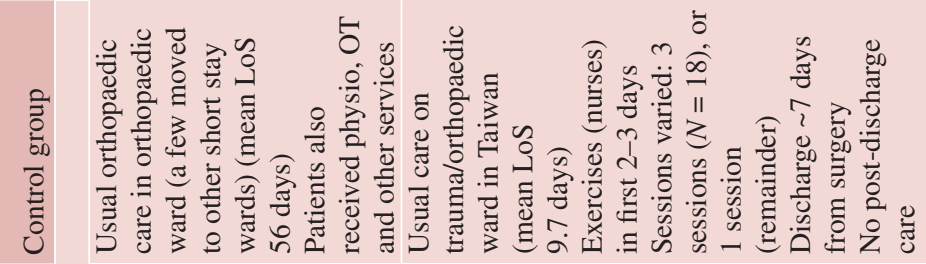

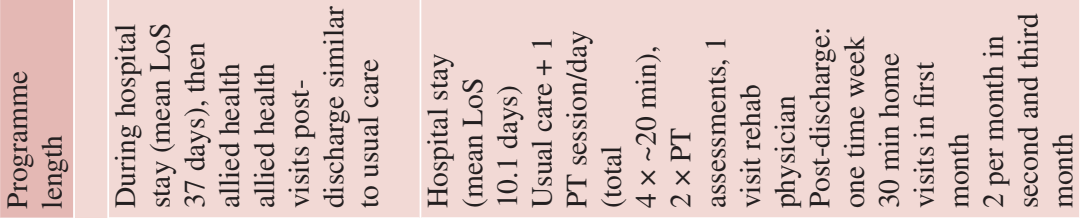

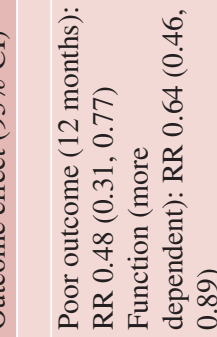
$\stackrel{\pi}{\simeq}$

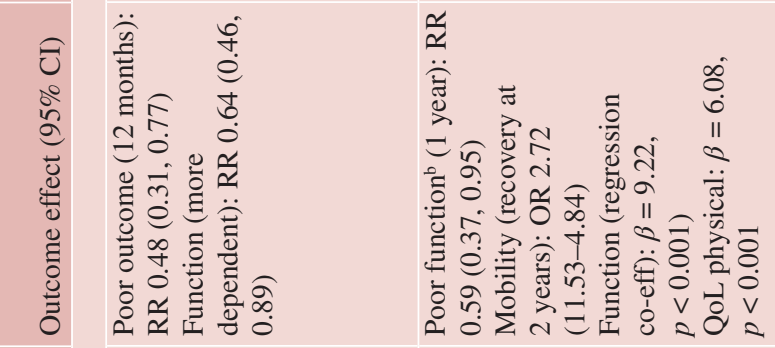

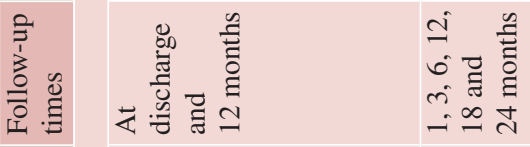

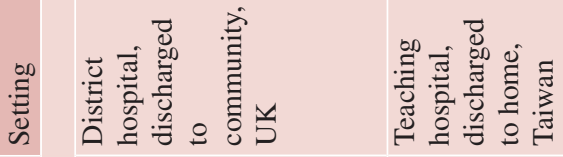

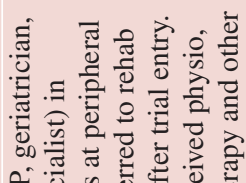

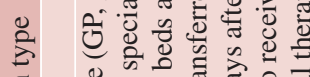

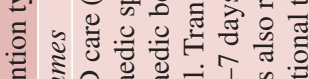

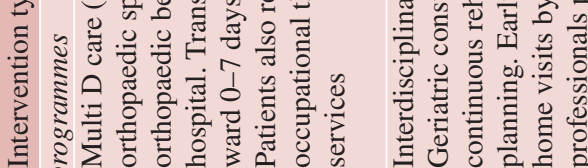

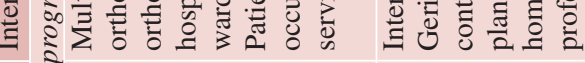

8

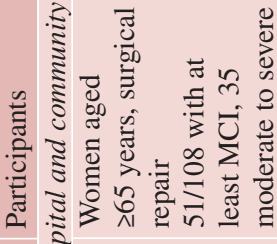
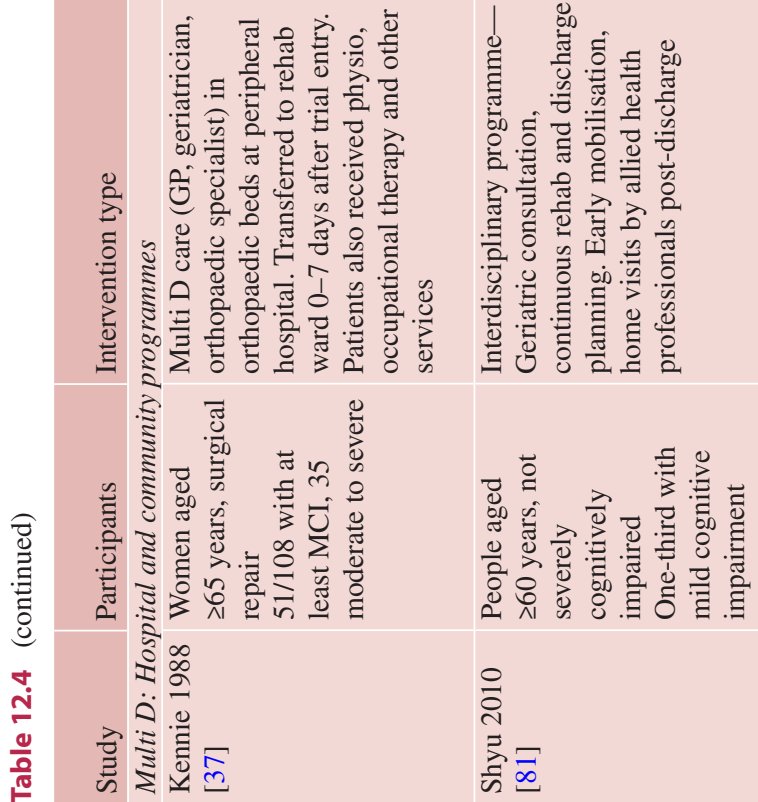


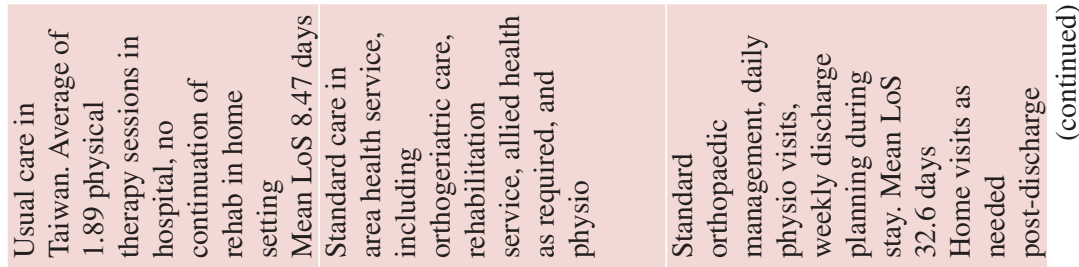

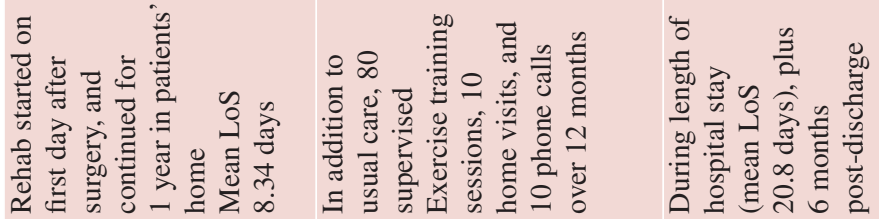

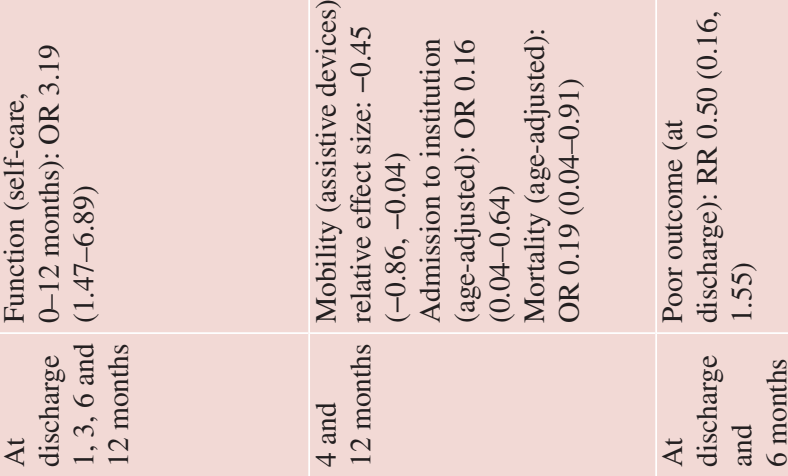

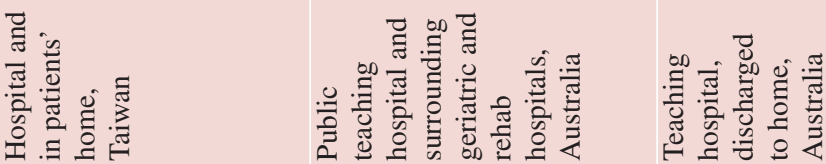

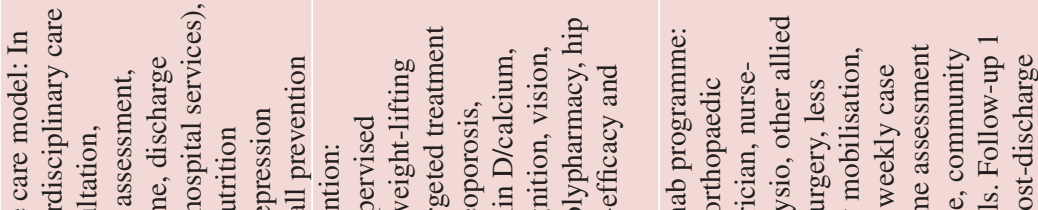

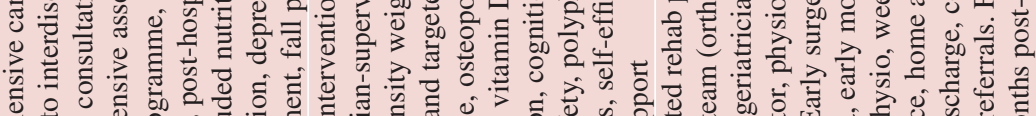

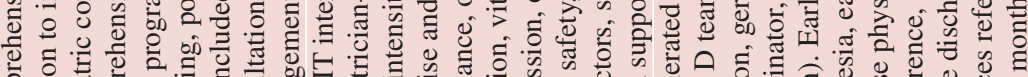

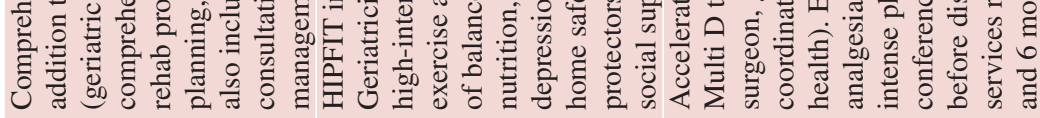

\begin{tabular}{|c|c|c|}
\hline 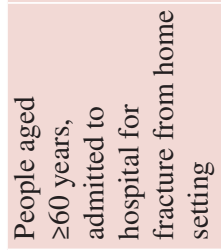 & 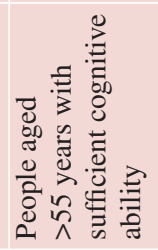 & 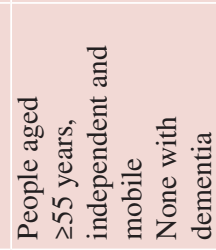 \\
\hline 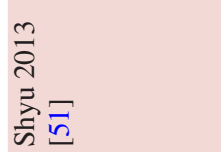 & 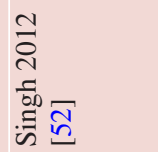 & 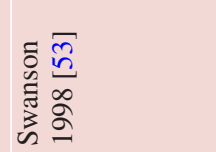 \\
\hline
\end{tabular}




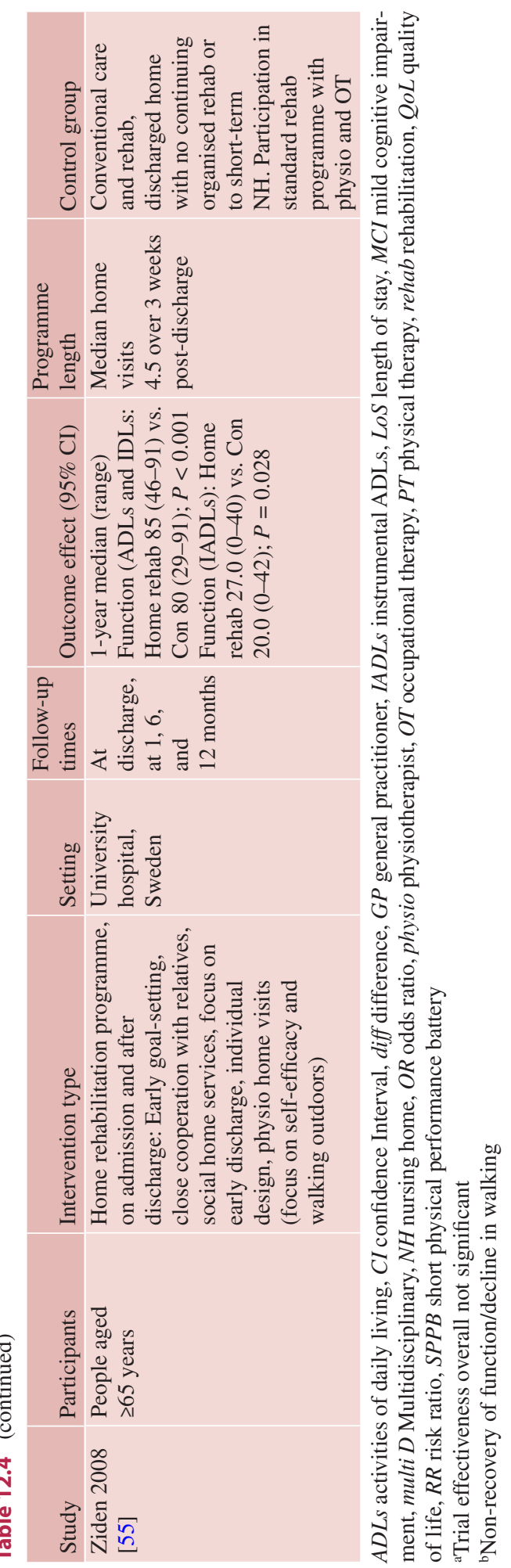




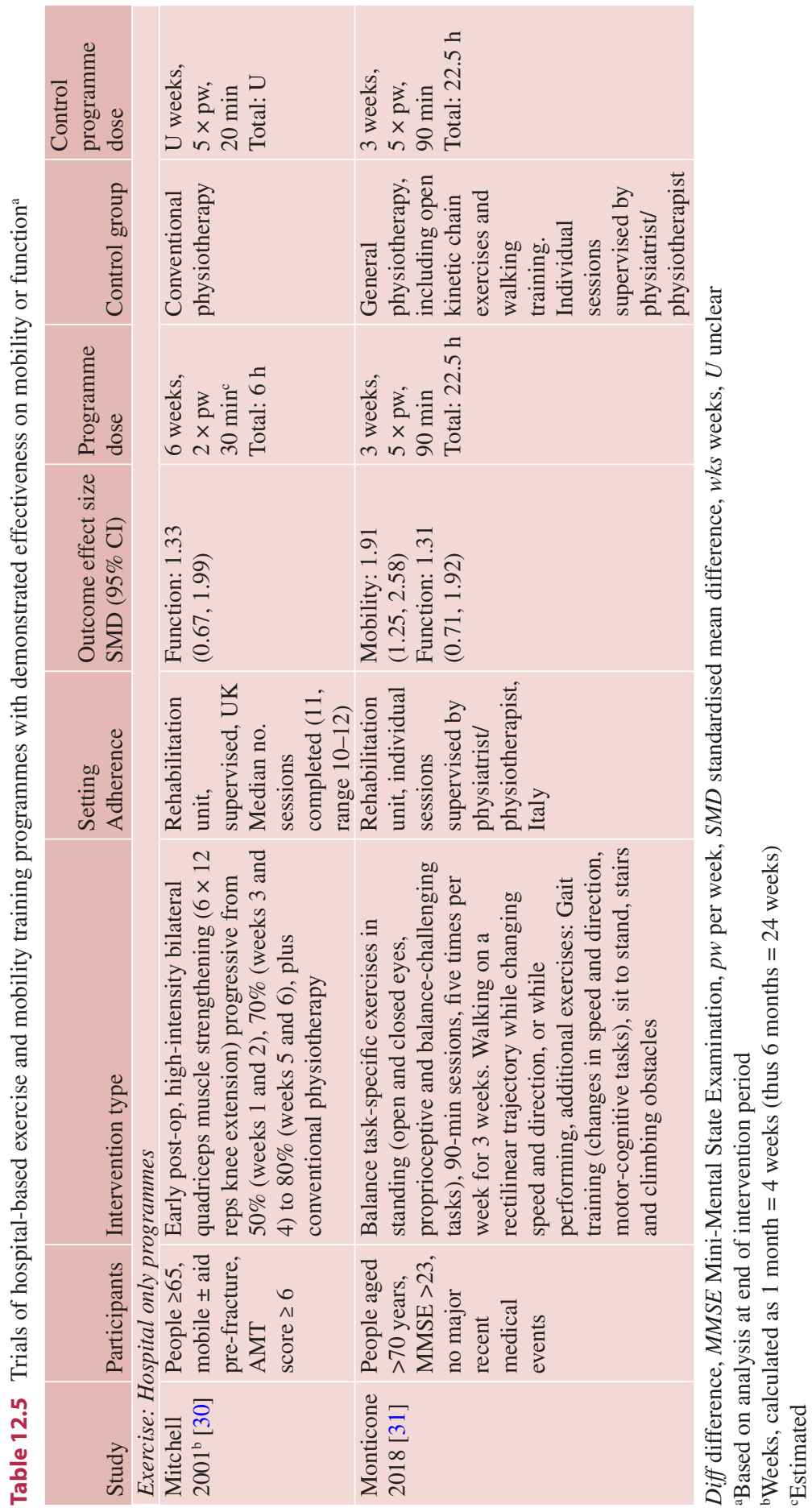




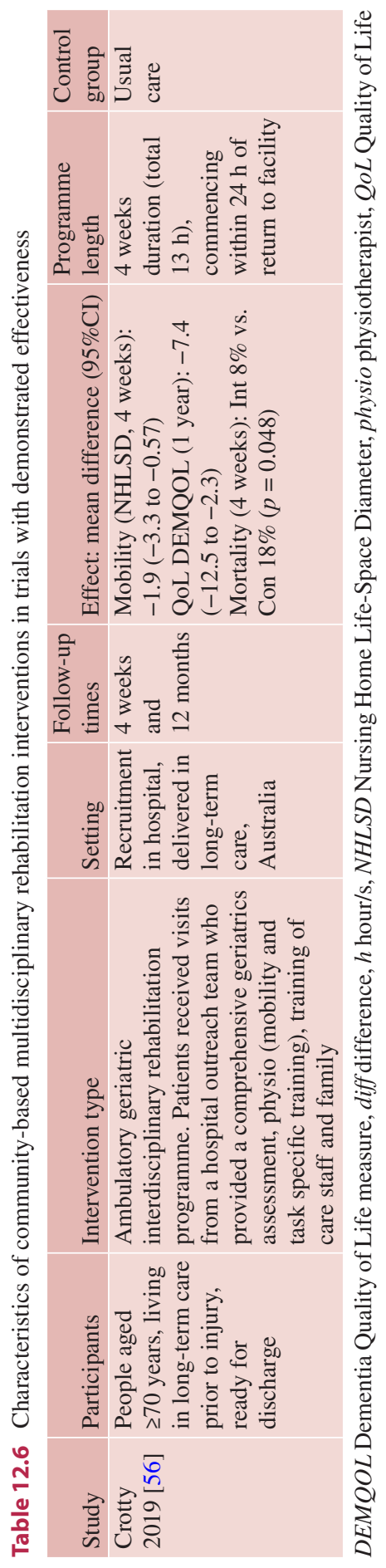




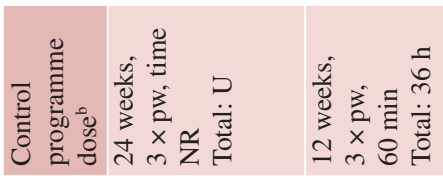

总

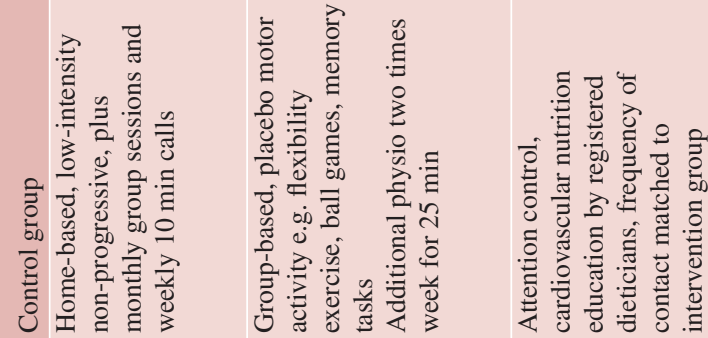

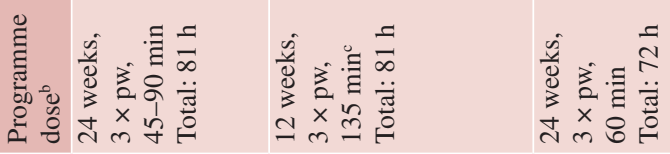

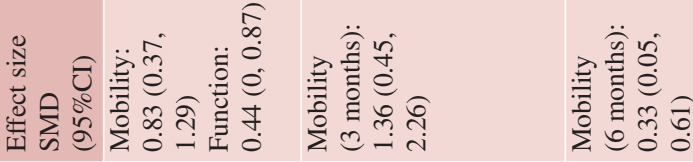

छे

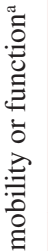

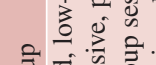

ธี

ळ

峞
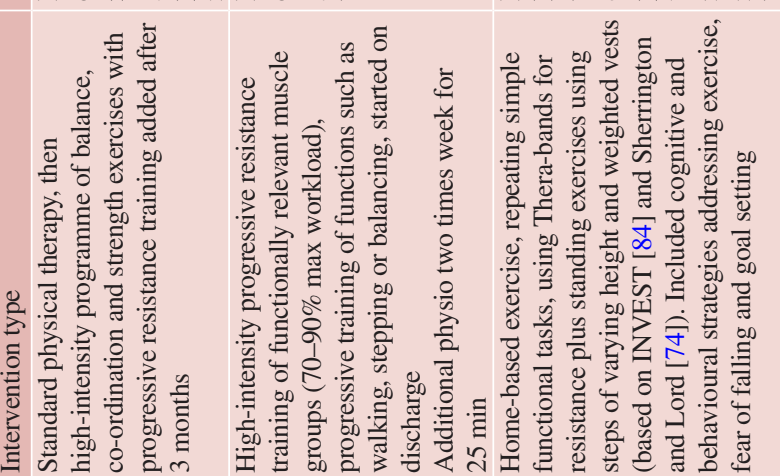

눈

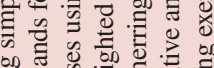

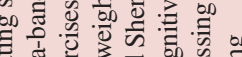

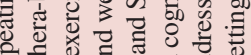
인

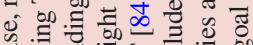

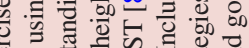

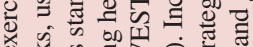

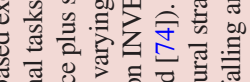

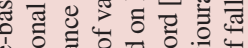

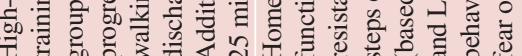

?

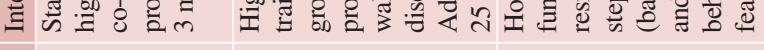

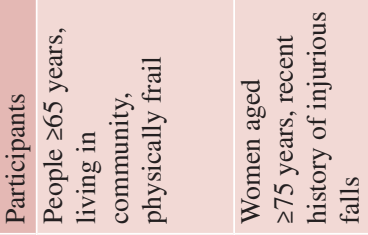

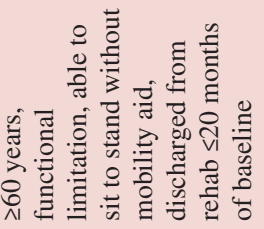




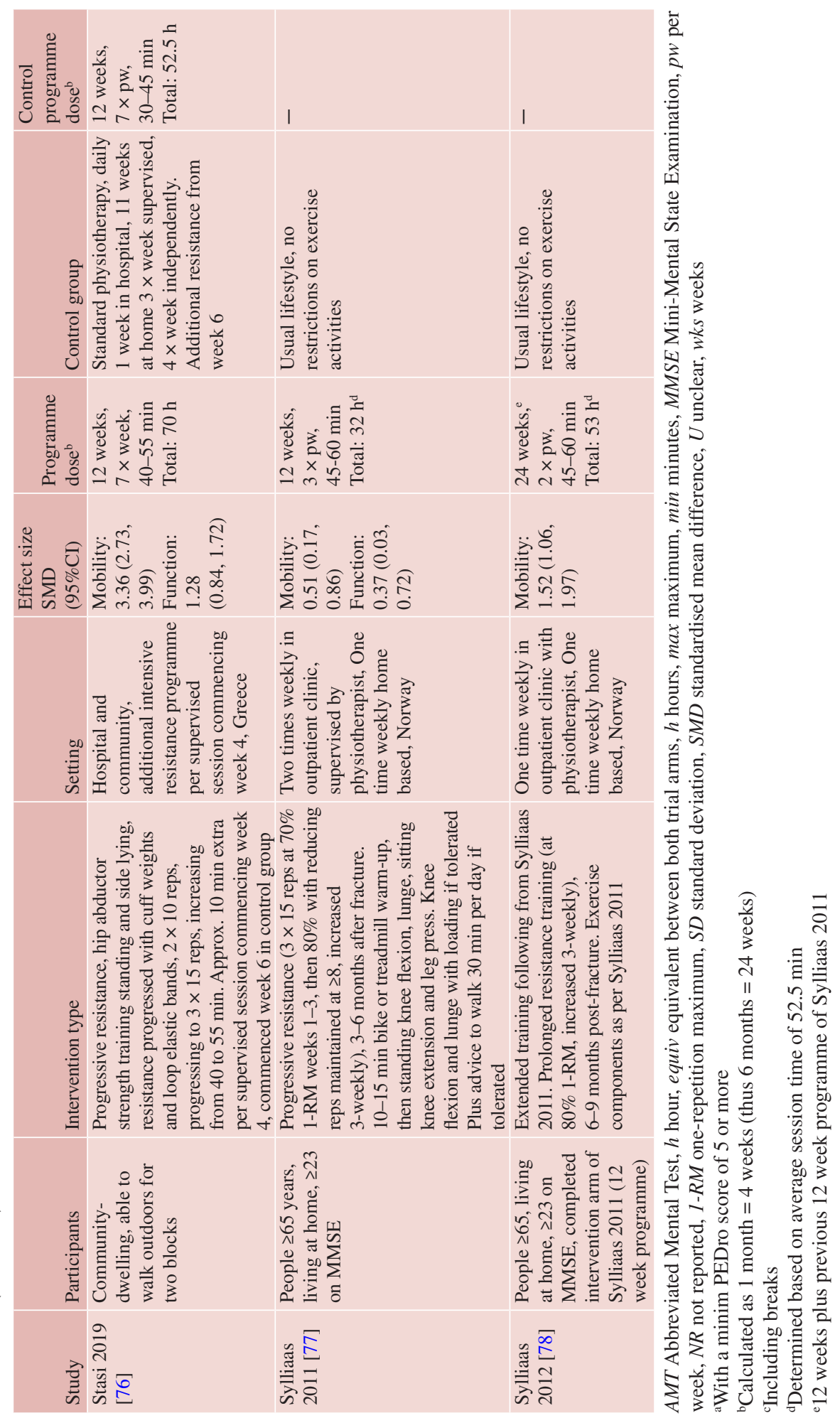


A more recent hospital-based trial has demonstrated effectiveness on a range of person-centred outcomes including mobility, function and quality of life at 1-year follow-up in comparison to standard orthopaedic care [40]. This programme provided comprehensive interdisciplinary care, early mobilisation and rehabilitation and also addressed psychosocial aspects of care through a focus on social situation and mental health.

Two exercise programmes that have demonstrated effectiveness in terms of improving mobility or function were delivered completely in an in-hospital (rehabilitation) setting (Table 12.5) [30, 31]. One study added progressive resistance training in the form of additional early post-operative, high-intensity bilateral quadriceps muscle strengthening to conventional physiotherapy. A significant improvement in the Elderly Mobility Scale, leg extensor power of the fractured leg and functional reach was reported at 16 weeks, which was 10 weeks after the end of the intervention [30]. The other programme which delivered a high dose of in-patient rehabilitation including supervised balance exercises (five times weekly for $90 \mathrm{~min}$, over 3 weeks), also demonstrated improvements in mobility and function compared to standard rehabilitation on discharge [31].

\subsubsection{Rehabilitation in the Community}

Six multidisciplinary interventions delivered across both hospital and community settings have demonstrated improvements in patient-centred outcomes in comparison to usual orthopaedic care (Table 12.4) [37, 50-53, 55]. Four of these trials were conducted in high income countries $[37,52,53,55]$ and two were conducted in Taiwan $[51,81]$. In general, multidisciplinary programmes that emphasise early assessment through comprehensive geriatric assessment with appropriate early surgery, early mobilisation, higher doses of mobility training and an emphasis on regaining functional independence are more effective. Multidisciplinary rehabilitation programmes (including those with a focus on multidisciplinary factors where specialist teams are not available) should also begin soon after hospital admission and continue for a long period, including after hospital discharge.

It remains unclear what is the best link between orthogeriatric services and hip fracture rehabilitation services to improve coordination for patients, but common governance structures, shared staff, shared information systems or formal arrangements for handovers are all options. These services should treat patients with dementia and delirium and also include patients who are living in, or will live in, residential aged care facilities. High intensity and prolonged multidisciplinary rehabilitation programmes (e.g. Singh et al. [52]) are effective for a selected group of people with hip fracture.

There is an emerging view that hip fracture rehabilitation programmes should also be available to people with significant dementia who live in long-term care, or at home, with severe disabilities. A recent trial of a four-week multidisciplinary programme delivered as a hospital outreach programme within long-term care demonstrated improvements in mobility at the end of the programme, which was not maintained over 12 months, but a small improvement in quality of life was observed at 12 months (Table 12.6) [56]. Whilst the programme was found not to be 
cost-effective, it demonstrates that improvements in patient-centred outcomes can be made after hospital discharge in a population living in long-term care.

As shown in Table 12.7, the exercise programmes that continued after discharge and were effective were programmes conducted over 12-24 weeks. At least for some individuals, there are benefits from exercise programmes delivered after discharge from hospital. One of the most effective programmes identified in our review of trials of exercise and mobility training programmes was implemented as twiceweekly sessions with a physiotherapist in an outpatient clinic for the first 3 months, then once weekly for a further 3 months (Table 12.7) [77, 78]. This was supplemented with exercises once a week at home. The exercise programme involved prolonged progressive resistance training, fitness warm-up and lower limb strength exercises, compared to a control group of the participant's usual lifestyle, without any restrictions placed on the amount or type of exercise undertaken. This programme significantly improved patient's mobility after 3 months [77], but the magnitude of the effect was even greater after 6 months [78]. While the strength of effect in this study may partly be due to a comparison against patients with no structured exercise programme, two other community-based programmes of progressive resistance training in small groups also demonstrated large effects in comparison to alternative programmes $[65,66]$. Another study has demonstrated that extra progressive resistance exercises in addition to a 12 -week standard daily physiotherapy programme can provide additional benefits for mobility and function [76].

However, long-term provision of exercise programmes through outpatient clinics for whole populations may not be feasible, even in developed countries, as this would require an enormous expansion of rehabilitation services with associated costs. Greater provision of community exercise options in liaison with health professionals may help to meet this gap, as has been recommended for people with neurological impairments [82].

\subsubsection{Rehabilitation in Low Resource Settings}

A trial conducted in Taiwan has demonstrated significant improvements in mobility and self-care extending to 2 years post-hip fracture from interdisciplinary rehabilitation programmes in comparison to usual care with no formal rehabilitation programme [81]. A further trial demonstrated additional benefits of a comprehensive care programme addressing nutrition, depression management and falls prevention in addition to interdisciplinary care in the same setting [51]. Whilst the same interdisciplinary care may not be possible in low resource and LMIC settings, rehabilitation programmes that address these principles using professionals with competencies in geriatrics, orthopaedics, physiotherapy, occupational therapy, nutrition, social work and psychology should be the aim.

Supervised exercise programmes may present access difficulties for people in remote locations or in low resource settings so home exercise, wider family involvement or tele-rehabilitation options may be required.

A home-based exercise programme of simple, functionally oriented tasks with minimal supervision had a moderate effect on improving physical function [68]. In 
this programme, a physical therapist taught the exercises and used cognitive and behavioural strategies to enhance attitudes and beliefs about the benefits of exercise and to overcome fear of falling during three home visits of $1 \mathrm{~h}$ (Table 12.7). Monthly telephone calls were also made by the therapists and an additional visit was provided if necessary. The participants were provided with a DVD of the programme to watch and a DVD player if necessary. Participants performed the exercises independently in their own home three times a week for 6 months, supported by a monthly telephone call from the physical therapist. The intervention also included a cognitive-behavioural component in order to improve adherence. A secondary analysis of this trial indicates that self-efficacy may partially mediate the effects of this intervention on longer term functional outcomes [83]. Whilst physical therapists may not be available in all resource settings, this trial demonstrates the potential effectiveness of homebased therapy. Alternative professionals with skills in physical therapy could provide training. Including caregivers in this training, where resources for watching a DVD and follow-up phone calls are not available, appears promising and warrants investigation.

\subsection{Rehabilitation and Cognitive Impairment}

Rehabilitation for people with dementia after hip fracture is complex. Approximately $40 \%$ of patients who sustain a hip fracture have dementia $[85,86]$. These patients have more complex care needs, with greater risks of complications, physical disabilities and social care requirements compared to people without dementia [87]. This is due to a number of factors. Firstly, people with dementia are often more disorientated in hospital environments, being more prone to delirium. They often have difficulty expressing problems of pain, nausea and dizziness which impact on physical performance. Many people with dementia have movement limitations, which when combined with hip fracture, makes simple tasks like learning how to use walking aids and equipment very difficult. They often have a critical relationship with informal caregivers (family/friends) which is strained after a hip fracture; greater considerations for supporting the patient-caregiver dyad may be required than for patients without dementia. Daily proactive geriatrician visits starting before or within $24 \mathrm{~h}$ of hip fracture surgery, with application of multiple types of treatment, has been demonstrated to reduce delirium occurrence by $36 \%$, and severe delirium by $60 \%$ [38]. Similar principles could be followed in LMIC settings with lesser intensity of inputs.

A number of research reports and guidelines recommend intervention with specific strategies including enhanced rehabilitation and care pathways to support recovery from hip fracture for people living with dementia. However, the evidence base for these is sparse. Five trials have investigated enhanced rehabilitation models for this population; evaluating strategies designed specifically for people with dementia following hip fracture surgery. These are larger trials of patients following hip fracture surgery which have presented data specifically for the subgroup of patient with cognitive impairment. These trials have tested two types of interventions: enhanced interdisciplinary inpatient rehabilitation and care models versus conventional 
inpatient rehabilitation and care models [43, 88, 89] and secondly, enhanced interdisciplinary inpatient and home-based rehabilitation and care models versus conventional rehabilitation and care models $[90,91]$. The characteristics of these trials and interventions are presented in Table 12.8. The enhanced models generally offer multidisciplinary programmes with greater intensity or length of programmes.

Table 12.8 Characteristics of trials of enhanced rehabilitation for people with dementia following hip fracture

\begin{tabular}{|c|c|c|c|c|c|}
\hline Study & $\begin{array}{l}\text { Setting } \\
\text { country }\end{array}$ & $\begin{array}{l}\text { Sample } \\
\text { size }\end{array}$ & PEDro & Characteristics of intervention & Comparator \\
\hline \multicolumn{6}{|c|}{ Hospital-based enhanced rehabilitation programmes } \\
\hline $\begin{array}{l}\text { Freter } \\
2017 \\
{[88]}\end{array}$ & $\begin{array}{l}\text { Hospital, } \\
\text { Canada }\end{array}$ & 283 & 4 & $\begin{array}{l}\text { Delirium-friendly care options } \\
\text { including: Orientation } \\
\text { strategies; night-time } \\
\text { sedation, analgesia, and } \\
\text { nausea; attention to catheter } \\
\text { removal and bowel } \\
\text { movements }\end{array}$ & $\begin{array}{l}\text { Standard recovery } \\
\text { programme }\end{array}$ \\
\hline $\begin{array}{l}\text { Stenvall } \\
2012 \\
{[89]}\end{array}$ & $\begin{array}{l}\text { Hospital, } \\
\text { Sweden }\end{array}$ & 64 & 6 & $\begin{array}{l}\text { MultiD team intervention: } \\
\text { Individual care planning, } \\
\text { monitoring for specific } \\
\text { common complications (falls, } \\
\text { delirium, bowel and bladder } \\
\text { care, sleep, pain, pressure } \\
\text { sores, physiological markers } \\
\text { and nutrition), early inpatient } \\
\text { rehabilitation with increased } \\
\text { staffing ratio }\end{array}$ & $\begin{array}{l}\text { Non-formalised and } \\
\text { inconsistent provision of } \\
\text { team working, } \\
\text { individualised care } \\
\text { planning, rehabilitation or } \\
\text { complication monitoring. } \\
\text { Prevention and treatment of } \\
\text { decubitus ulcers, pain } \\
\text { management and basic } \\
\text { care, but no dietitian review }\end{array}$ \\
\hline $\begin{array}{l}\text { Uy } 2008 \\
{[43]}\end{array}$ & $\begin{array}{l}\text { Hospital, } \\
\text { Australia }\end{array}$ & & 8 & $\begin{array}{l}\text { Early mobility and self-care } \\
\text { (nurse delivered). Target of } \\
\text { twice-daily physiotherapy } \\
\text { with greater multiD team } \\
\text { involvement in mobility and } \\
\text { enablement }\end{array}$ & $\begin{array}{l}\text { Standard recovery } \\
\text { programme }\end{array}$ \\
\hline \multicolumn{6}{|c|}{ Hospital- and community-based enhanced rehabilitation programmes } \\
\hline $\begin{array}{l}\text { Huusko } \\
2000 \\
{[90]}\end{array}$ & $\begin{array}{l}\mathrm{H} \& \mathrm{C} \\
\text { Finland }\end{array}$ & 141 & 7 & $\begin{array}{l}\text { Enhanced multi D team } \\
\text { rehabilitation including two } \\
\text { times daily physiotherapy, } \\
\text { multiD team meetings and } \\
\text { improved communication } \\
\text { across the team and with } \\
\text { patients. Plus discharge } \\
\text { planning and ten home-based } \\
\text { physiotherapy sessions }\end{array}$ & $\begin{array}{l}\text { Standard recovery } \\
\text { programme. All } \\
\text { participants encouraged to } \\
\text { mobilise on the first } \\
\text { post-operative day. No } \\
\text { further information } \\
\text { provided }\end{array}$ \\
\hline $\begin{array}{l}\text { Shyu } \\
2012 \\
{[91]}\end{array}$ & $\begin{array}{l}\mathrm{H} \& \mathrm{C} \\
\text { Taiwan }\end{array}$ & 160 & 6 & $\begin{array}{l}\text { Enhanced multiD team, two } \\
\text { times daily physio, multiD } \\
\text { team meetings, improved } \\
\text { communication and } \\
\text { individualise care. Plus } \\
\text { individualised discharge } \\
\text { planning, three home-based } \\
\text { physio and eight home-based } \\
\text { nurse visits }\end{array}$ & $\begin{array}{l}\text { Standard recovery } \\
\text { programme. Inpatient } \\
\text { rehabilitation consisted of } \\
3 \text { physiotherapy sessions, } \\
\text { and no in-home } \\
\text { rehabilitation. No further } \\
\text { information provided }\end{array}$ \\
\hline
\end{tabular}

$C$ community, $H$ hospital, $H \& C$ hospital and community, MultiD multidisciplinary, physio physiotherapy 


\subsubsection{Enhanced Interdisciplinary Inpatient Rehabilitation and Care}

The clinical outcomes of enhanced inpatient rehabilitation care compared to conventional care are summarised in Table 12.9. It appears there was no benefit of enhanced interdisciplinary inpatient rehabilitation over conventional care for outcomes including personal ADL independence at four-month or 12-month followup, walking independence without an aid or assistance at four-month or 12-month

Table 12.9 Clinical outcomes of enhanced rehabilitation interventions for people with dementia following hip fracture

\begin{tabular}{|c|c|c|c|c|}
\hline Outcome measure & $\begin{array}{l}\text { Time- } \\
\text { point } \\
\text { (months) }\end{array}$ & Participants & Study & Outcome OR/MD (95\% CI) \\
\hline \multicolumn{5}{|c|}{ In-patient enhanced rehabilitation programmes } \\
\hline \multirow{2}{*}{$\begin{array}{l}\text { Personal activities } \\
\text { of daily living } \\
\text { independence }\end{array}$} & 4 & 54 & Stenvall 2012 [89] & OR $4.14(0.40-42.66)$ \\
\hline & 12 & 47 & Stenvall $2012[89]$ & OR $4.62(0.18-119.63)$ \\
\hline \multirow{2}{*}{$\begin{array}{l}\text { Walking } \\
\text { independence } \\
\text { without an aid or } \\
\text { assistance }\end{array}$} & 4 & 54 & Stenvall 2012 [89] & OR $7.63(0.83-70.53)$ \\
\hline & 12 & 47 & Stenvall 2012 [89] & OR $7.20(0.74-70.42)$ \\
\hline Mortality & Discharge & 151 & $\begin{array}{l}\text { Freter 2016; } \\
\text { Stenvall 2012; Uy } \\
2008[43,88,89]\end{array}$ & OR $0.62(0.22-1.74)$ \\
\hline $\begin{array}{l}\text { Hospital Length of } \\
\text { Stay }\end{array}$ & Discharge & 141 & $\begin{array}{l}\text { Freter 2016; } \\
\text { Stenvall } 2012 \\
{[88,89]}\end{array}$ & $\mathrm{MD}-3.24(-8.75$ to 2.26$)$ \\
\hline \multicolumn{5}{|c|}{ In-patient and community-based enhanced rehabilitation programmes } \\
\hline \multirow[t]{2}{*}{ Mortality } & 3 & 184 & $\begin{array}{l}\text { Huusko } 2000 ; \\
\text { Shyu } 2012[90,91]\end{array}$ & OR $1.20(0.36-3.93)$ \\
\hline & 12 & 177 & $\begin{array}{l}\text { Huusko 2000; } \\
\text { Shyu } 2012[90,91]\end{array}$ & OR 1.07 (0.47-2.45) \\
\hline \multirow[t]{2}{*}{$\begin{array}{l}\text { Requirement of } \\
\text { institutional care }\end{array}$} & 3 & 184 & $\begin{array}{l}\text { Huusko 2000; } \\
\text { Shyu } 2012[90,91]\end{array}$ & OR $0.46(0.22-0.95)$ \\
\hline & 12 & 177 & $\begin{array}{l}\text { Huusko 2000; } \\
\text { Shyu } 2012[90,91]\end{array}$ & OR $0.90(0.40-2.03)$ \\
\hline \multirow{8}{*}{$\begin{array}{l}\text { Regained their } \\
\text { pre-fracture } \\
\text { walking capability } \\
\text { ADL performance }\end{array}$} & 3 & 43 & Shyu 2012 [91] & OR $5.10(1.29-20.17)$ \\
\hline & 12 & 36 & Shyu 2012 [91] & OR $58.33(3.04-1118.19)$ \\
\hline & 24 & 30 & Shyu 2012 [91] & OR $3.14(0.68-14.50)$ \\
\hline & 3 & 43 & Shyu 2012 [91] & MD $18.81(9.40-28.22)$ \\
\hline & 12 & 36 & Shyu 2012 [91] & MD $25.40(10.89-39.91)$ \\
\hline & 24 & 30 & Shyu 2012 [91] & MD 7.92 ( -9.88 to 25.72$)$ \\
\hline & 12 & 36 & Shyu 2012 [91] & OR $0.20(0.01-4.47)$ \\
\hline & 24 & 30 & Shyu 2012 [91] & OR 0.77 (0.16-3.74) \\
\hline
\end{tabular}

$C I$ confidence interval $M D$ mean difference $O R$ odds ratio 
follow-up or the number of drugs prescribed on discharge. Similarly, there were no differences in outcomes for mortality or hospital length of stay for enhanced inpatient rehabilitation models over conventional care.

There was no benefit of an enhanced inpatient rehabilitation programme over conventional rehabilitation for complications including pneumonia, pressure ulcers, postoperative fracture or whether participants were living in care facilities at 4 months or 12 months. However, there was a reduction in the enhanced interdisciplinary rehabilitation care model group for complications including urinary tract infection, nutritional problems, recurrent falls and post-operative delirium. Freter and colleagues also reported greater cognitive function for those who received the enhanced intervention 5 days post-operatively compared to conventional rehabilitation [88].

\subsubsection{Enhanced Interdisciplinary Inpatient and Home-Based Rehabilitation}

The clinical outcomes of enhanced rehabilitation inpatient and home-based rehabilitation care compared to conventional care are summarised in Table 12.9. Findings suggest that enhanced inpatient and community-based interventions for people with cognitive impairment provide promising early outcomes, but do not differ to conventional rehabilitation models longer term. Whilst people allocated enhanced interdisciplinary rehabilitation were less likely to be living in institutional care at 3 months, this was less certain at 12 months. One trial conducted in Taiwan reported that patients who received enhanced rehabilitation strategies until 3 months postdischarge had improvements in regaining pre-fracture walking levels and better ADL performance at 3 and 12 months, but did not differ from conventional rehabilitation at 24 months [91]. The evidence suggests no benefit of the enhanced inpatient and home-based intervention for outcomes including frequency of hospital admissions, attendance at the emergency room/accident and emergency, incidence of falls or mortality at 4 or 12 months post-operatively.

Whilst the current evidence-base provides a basis, the data remain very low in quality due to the small number of participants and the serious risk of bias in trial designs. The evidence underpinning the rehabilitation of people with dementia following hip fracture is based on subgroup analyses of randomised controlled trials of people with and without cognitive impairment who have a hip fracture. Consequently, the evidence-base is underpowered. No data were provided on behaviour, quality of life, pain or complications. No trials have investigated interventions which have been specifically designed for people with cognitive impairment. It remains unclear whether rehabilitation models are more effective if they include dementia-focused interventions such as provision of cues, reminiscence therapy, the adoption of familiarised routines or the use of assistive technologies. These are areas of research priority. Following this, it is hoped that health professionals will be able to be more evidence-based in addressing the complex care needs for this subgroup of the hip fracture population. 
Nevertheless, it is clear that patients with cognitive impairment also benefit from rehabilitation approaches and these patients should not be excluded from rehabilitation following hip fracture.

\subsection{Psychosocial Factors and Rehabilitation}

Within the WHO ICF framework, psychosocial factors can be environmental or personal "contextual" factors (e.g. social support, self-efficacy, fear of falling) or psychological "body function" factors (e.g. mental health) that interact with health conditions to impact on a person's functioning and recovery. Psychosocial factors are predictors of hip fracture recovery and their role in functional recovery after hip fracture has been acknowledged as important [92]. Depressive symptoms post hip fracture increase the likelihood of poorer mobility, function and psychological outcomes [92-94]. Fear of falling is common in people with hip fracture and is associated with poorer recovery, decreased mobility, anxiety and falls-related selfefficacy [95-97]. Social support and caregiver responses also appear to play a dynamic role in recovery $[98,99]$. However, the relationships between psychosocial factors, rehabilitation programmes and outcomes are complex and inadequately understood.

Clinicians need to support patients' adjustment to residual disability when providing rehabilitation to people with fragility fractures. Hip fractures are common and many older people in the community hold the fear that a hip fracture will precipitate a move into a residential aged care facility. In an Australian time-trade-off study, $80 \%$ of community-dwelling women at risk of hip fracture said they would rather die rather than suffer a hip fracture requiring relocation into a residential aged care facility [100]. The participants of this study commonly believed that they were living on "borrowed time" having survived beyond usual life expectancy. They perceived any threat to their ability to live independently in the community as potentially catastrophic.

When individuals experience changes in their health states, they often alter their internal standards, their values and concept of quality of life which is sometimes described as a "response shift" [101]. After a hip fracture, many people are left walking with an aid, with restrictions in the use of public transport, hobbies and roles, thus a significant loss of quality of life may occur. Maximising functional recovery is important but providing adequate support for older people to make "response shifts" and adjustments and to identify ways to compensate for changes is equally important e.g. by acknowledging losses in mobility but providing access to alternatives.

A randomised controlled trial of a home-based hip fracture rehabilitation intervention which included psychological strategies improved mobility outcomes for patients $[68,83]$. The study found that the intervention protected against the loss of self-efficacy. As self-efficacy appears to play a crucial role in maintaining exercise long-term, a focus on self-efficacy in hip fracture interventions may mean that 
patients are more likely to continue activity independently [83]. Qualitative studies indicate that hip fracture patients recognise the importance of their own psychological outlook and the need for social support in their recovery. Support from health professionals provides not only information and exercises but also emotional and motivational support and confidence boosting. Support from informal caregivers, family and friends is also seen as invaluable to help with ADLs, emotional support, encouragement and companionship [95, 102, 103]. Thus, inclusion of psychological and social interventions in hip fracture rehabilitation programmes is likely to be beneficial. However, the specifics of how to best design such programmes to improve outcomes are yet unclear [92].

\subsection{Delivery of Rehabilitation Following Hip Fracture in LMICs}

The prevalence of hip fracture is expected to increase dramatically in middleincome countries in Asia and Latin America, presenting a major challenge to rehabilitation care in coming years. By the year 2050, around 30\% of the world's hip fractures will occur in Asia, mostly in China and India. Although the rate of increase in incidence of hip fracture has been attenuated in Hong Kong and Taiwan, it has markedly increased for almost all age groups in both genders in mainland China [104]. India lacks a systematic data registry for fragility hip fractures, but a report in 2004 estimated an annual prevalence of 600,000 hip fractures, which will substantially increase, since the population over 60 years in 2026 will reach nearly 170 million people [105].

Overall hip fractures in the Latin America region will increase by $700 \%$ in the population 65 and over with an estimated cost of $\$ 13$ billion [106]. Based on population ageing estimates in Brazil the increase of hip fracture prevalence is estimated to be nearly $250 \%$ between 2015 and 2040 [107]. In Mexico, another highly-populated country, hip fracture rate estimates are sparse, but one study showed similar rates to southern countries in Europe [108].

The impact of hip fractures is unfavourable for patients and their families in LMICs as many do not have health care systems which are able to deliver integrated services including rehabilitation. Barriers exist in terms of human resources capability, infrastructure, cultural and social influences and environmental context.

While research on barriers and facilitators for rehabilitation following hip fracture is still scarce in most LMICs, Tables 12.10 and 12.11 describe known barriers to prompt in-hospital and community rehabilitation following hip fracture surgery in LMICs. 
Table 12.10 Barriers to prompt in-hospital rehabilitation following hip fracture surgery in LMICs

\begin{tabular}{|c|c|c|}
\hline Barrier category & Items & Examples \\
\hline $\begin{array}{l}\text { Environmental } \\
\text { context and } \\
\text { resources }\end{array}$ & 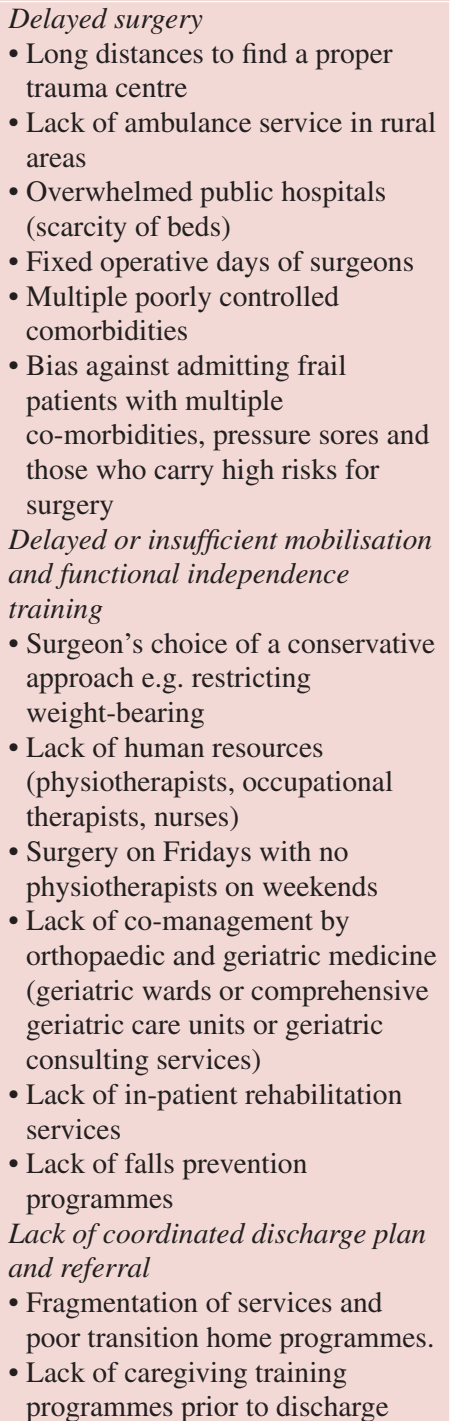 & 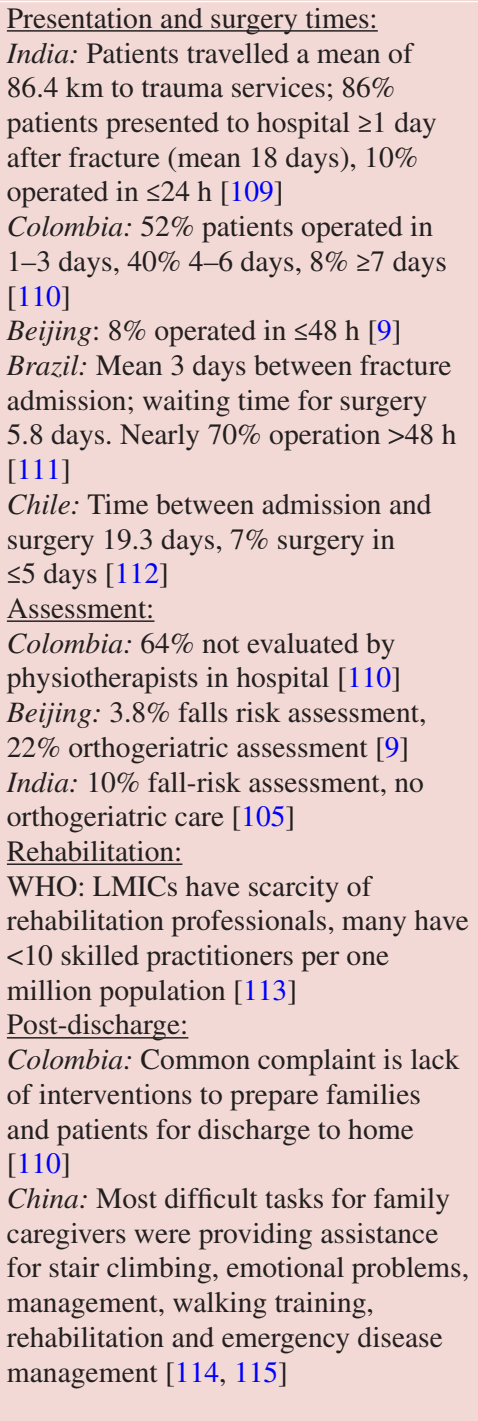 \\
\hline $\begin{array}{l}\text { Cultural and } \\
\text { social }\end{array}$ & $\begin{array}{l}\text { Delayed surgery } \\
\text { - Lack of knowledge or information } \\
\text { of family members about the } \\
\text { urgency of a fall-injury event } \\
\text { - Patients' beliefs in traditional bone } \\
\text { healers and aversion to surgical } \\
\text { interventions } \\
\text { Burden of family caregivers } \\
\text { - Low socioeconomic background } \\
\text { and social vulnerability }\end{array}$ & $\begin{array}{l}\text { India: Most patients and relatives had } \\
\text { no knowledge of the consequences of } \\
\text { hip fracture injury in older people } \\
\text { [109] } \\
\text { Internationally: Family financial } \\
\text { overload related to medical } \\
\text { appointments, private rehabilitation } \\
\text { and transport [115] }\end{array}$ \\
\hline
\end{tabular}


Table 12.10 (continued)

\begin{tabular}{|c|c|c|}
\hline Barrier category & Items & Examples \\
\hline $\begin{array}{l}\text { Human } \\
\text { resources } \\
\text { capability }\end{array}$ & $\begin{array}{l}\text { Delayed surgery } \\
\text { - Untrained health care } \\
\text { professionals in primary and } \\
\text { secondary care services } \\
\text { Delayed mobilisation and functional } \\
\text { independence training } \\
\text { - Evidence-based recommendation } \\
\text { of early mobilisation, pain and } \\
\text { delirium management, and fall risk } \\
\text { assessment is not in routine care } \\
\text { yet } \\
\text { - Lack of training for nurses, } \\
\text { physiotherapists and occupation } \\
\text { therapists } \\
\text { - Lack of a coordinated } \\
\text { multidisciplinary approach } \\
\text { - Failure to assess frailty and } \\
\text { patients' previous and ongoing } \\
\text { cognitive status with consequently } \\
\text { limited access to appropriate and } \\
\text { timely rehabilitation interventions } \\
\text { - Poor attitude or bias against } \\
\text { patients who are very old and/or } \\
\text { with cognitive decline due to } \\
\text { belief that outcomes will be } \\
\text { disappointing and rehabilitation } \\
\text { ineffective, thereby hopeless }\end{array}$ & $\begin{array}{l}\text { India and China: Lack of falls } \\
\text { assessment significant gap in care } \\
\text { pathway for hip fracture in hospitals } \\
{[105,109]} \\
\text { Brazil: Falls are the main cause of } \\
\text { death during the first } 30 \text { days after } \\
\text { surgery, representing } 43.5 \% \text { of deaths } \\
\text { [116] }\end{array}$ \\
\hline
\end{tabular}

\subsubsection{Key Evidence-Based Recommendations and Their Implementation in LMICs}

Implementation of evidence-based recommendations in LMICs is challenging but should not be interpreted as a wasted effort. Table 12.12 lists some key evidencebased recommendations and suggestions for implementation of in-hospital rehabilitation and community rehabilitation following hip fracture surgery in LMICs, based on expert opinion. Suggestions with limited formal evidence but apparent face validity include involving families as partners early and explicitly including them in the care plan and ensuring that ward nurses and therapists jointly commit to delivering the mobility goals.

With limited infrastructure (rehabilitation units and trained therapists) and rapidly growing demand, disruptive approaches to rehabilitation are needed in LMICs. WHO's Integrated Care for Older People (ICOPE) programme for older people is a community-based primary care health ageing approach which focuses on ways of optimising a community dwelling older person's function. However, many of the tools and resources provided in this programme allow community workers to design 
Table 12.11 Barriers to rehabilitation in the community in LMICs

\begin{tabular}{|c|c|c|}
\hline Barrier category & Items & Examples \\
\hline $\begin{array}{l}\text { Environmental } \\
\text { context and } \\
\text { resources }\end{array}$ & $\begin{array}{l}\text { Lack of comprehensive geriatric care services to } \\
\text { conduct structured, systematic inter-disciplinary } \\
\text { geriatric assessment (including physical and } \\
\text { mental health, function and social support } \\
\text { condition) } \\
\text { Ineffective and under-utilised referral pathways } \\
\text { to rehabilitation after hospital discharge } \\
\text { No timely and affordable rehabilitation } \\
\text { interventions (long waiting times) } \\
\text { Lack of adequate service network and } \\
\text { rehabilitation facilities to meet different } \\
\text { rehabilitation needs (inpatient, outpatient and } \\
\text { homecare) } \\
\text { Lack of rehabilitation staff, particularly } \\
\text { occupational therapists } \\
\text { Poor adherence due to lack of transportation, } \\
\text { inability of caregiver to take time off work and } \\
\text { long distances to rehabilitation facilities } \\
\text { Inadequate resources (assistive technologies and } \\
\text { devices) } \\
\text { Lack of standardised protocols for post-acute care } \\
\text { Lack of accessibility and poor neighbourhoods } \\
\text { preventing independence in walking outdoors }\end{array}$ & $\begin{array}{l}\text { Taiwan: Community- } \\
\text { based home care services } \\
\text { are designed for people } \\
\text { with long-term care needs } \\
\text { and are mainly skilled } \\
\text { nursing care-doesn't } \\
\text { completely fit post-acute } \\
\text { care needs [117] } \\
\text { Brazil: } 70 \% \text { patients had } \\
\leq 3 \text { months rehabilitation } \\
\text { after surgery, mostly one } \\
\text { time weekly; } 17 \% \text { had } \\
\text { home care rehabilitation } \\
\text { [118] } \\
\text { India and Brazil: } \\
\text { Physiotherapy largely } \\
\text { delivered in acute care, } \\
\text { access difficult in public } \\
\text { services. Most families } \\
\text { don't have resources to } \\
\text { pay for private services } \\
\text { Brazil: Majority of } \\
\text { rehabilitation } \\
\text { programmes based on the } \\
\text { model of traditional } \\
\text { clinic- and hospital-based } \\
\text { settings, failing to reach } \\
\text { frail older adults at home }\end{array}$ \\
\hline $\begin{array}{l}\text { Cultural and } \\
\text { social influences }\end{array}$ & $\begin{array}{l}\text { Negative social representation of old age } \\
\text { Cultural attitudes and beliefs toward disability in } \\
\text { old age, older adults have to be convinced that } \\
\text { mobility related problems are treatable and falls } \\
\text { can be prevented }\end{array}$ & $\begin{array}{l}\text { Brazil: Negative } \\
\text { connotations of age and a } \\
\text { sense of passivity were } \\
\text { experienced by older } \\
\text { adults after hip fracture } \\
\text { surgery, leading to } \\
\text { immobility and inactivity } \\
\text { (qualitative study) [95] }\end{array}$ \\
\hline $\begin{array}{l}\text { Human resources } \\
\text { capability }\end{array}$ & $\begin{array}{l}\text { Lack of training on systematic inter disciplinary } \\
\text { geriatric assessment } \\
\text { Lack of training on fragility fractures, frailty, } \\
\text { sarcopenia and bone health } \\
\text { Lack of training on evidence-based exercise } \\
\text { approach (balance and resistance training and } \\
\text { falls prevention) } \\
\text { Lack of fracture liaison services with a } \\
\text { multidisciplinary care approach }\end{array}$ & \\
\hline
\end{tabular}

aSecondary data from a clinical trial

individualised programmes for older people. Available as an app or on-line some of these practical approaches (e.g. on nutrition, polypharmacy, carer support) could be helpful for primary care workers once a patient with a hip fracture returns home (available from www.who.int/ageing/health-systems/icope/en/). 
Table 12.12 Implementation strategies in LMICs, based on expert opinion

\begin{tabular}{|c|c|}
\hline $\begin{array}{l}\text { In-hospital rehabilitation evidence- } \\
\text { based recommendations }\end{array}$ & Suggested implementation strategies \\
\hline $\begin{array}{l}\text { 1. Comprehensive screening and } \\
\text { assessment of older adults } \\
\text { immediately after admission on } \\
\text { trauma or orthopedic ward by a } \\
\text { skilled trained nurse }\end{array}$ & $\begin{array}{l}\text { - Make available a short, feasible, reliable and valid tool } \\
\text { kit for nurses to screen for frailty and delirium } \\
\text { - Identify poor nutritional status and dysphagia as early } \\
\text { as possible } \\
\text { - Take actions to overcome modifiable risk factors } \\
\text { - Where orthogeriatric care is not routine, nurses should } \\
\text { request a geriatric consultation for complex cases } \\
\text { - Where rehabilitation team members are available } \\
\text { (physiatrist, physiotherapist, occupational therapist } \\
\text { and speech therapy) a multidisciplinary assessment } \\
\text { should be conducted }\end{array}$ \\
\hline $\begin{array}{l}\text { 2. Physiotherapy approach } \\
\text { immediately after admission }\end{array}$ & $\begin{array}{l}\text { If surgery is delayed, immediate physiotherapy should } \\
\text { begin to prevent muscle strength decline of the } \\
\text { non-fractured leg, avoid respiratory problems (such as } \\
\text { pneumonia), and pressure ulcers }\end{array}$ \\
\hline $\begin{array}{l}\text { 3. Include caregivers in the care } \\
\text { planning from time of ward } \\
\text { admission }\end{array}$ & $\begin{array}{l}\text { Caregivers may request to stay with older adults in the } \\
\text { ward and should be included in the care plan. Train } \\
\text { caregivers to identify signs of complications (such as } \\
\text { delirium, swallowing problems, falls, pain) and help } \\
\text { with daily basic activities, this can build and strengthen } \\
\text { their skills for hospital discharge. Empower caregivers } \\
\text { as co-participants in care during hospital stay }\end{array}$ \\
\hline $\begin{array}{l}\text { 4. Surgical planning should target } \\
\text { early weight-bearing }\end{array}$ & $\begin{array}{l}\text { Weight-bearing restriction in the post-operative phase } \\
\text { should be avoided since it limits what a patient can } \\
\text { achieve in terms of mobility and functional } \\
\text { independence. Effective surgery allows early weight } \\
\text { bearing }\end{array}$ \\
\hline $\begin{array}{l}\text { 5. Early mobilisation after surgery } \\
\text { ( } 24 \mathrm{~h} \text { after surgery) }\end{array}$ & $\begin{array}{l}\text { Unless medically or surgically contraindicated, } \\
\text { physiotherapists and/or nurses should sit patients out of } \\
\text { bed, and walk as early as possible } \\
\text { Care plans delivered co-jointly by nurses and } \\
\text { physiotherapists can promote patients' mobility over the } \\
\text { entire day }\end{array}$ \\
\hline $\begin{array}{l}\text { 6. Early post-operative goal-directed } \\
\text { mobilisation practice with balance } \\
\text { and functional exercises }\end{array}$ & $\begin{array}{l}\text { Begin progressive resistance exercises, weight-bearing } \\
\text { exercises (unless contra-indicated) and balance } \\
\text { exercises during hospital stay, starting as early as } \\
\text { possible } \\
\text { Some services in LMICs are based on early discharge } \\
\text { from hospital to rehabilitation at home ("hospital at } \\
\text { home"). Harness informal and formal caregiving } \\
\text { training and use technology but be aware how to } \\
\text { achieve the best results when relying on families as } \\
\text { therapists in LMICs, particularly when education levels } \\
\text { are low }\end{array}$ \\
\hline $\begin{array}{l}\text { 7. Fall risk assessment during } \\
\text { hospital stay }\end{array}$ & $\begin{array}{l}\text { Provide falls risk assessments to patients/caregivers } \\
\text { with educational information and referral to community } \\
\text { services after discharge }\end{array}$ \\
\hline 8. Discharge planning & $\begin{array}{l}\text { Nurses should prepare caregivers for the level of care at } \\
\text { home. Educational materials and practical carer training } \\
\text { should be provided where possible [119] }\end{array}$ \\
\hline
\end{tabular}


Access to rehabilitation with the associated opportunity to maximise function and quality of life is increasingly recognised as a human right. Efforts to increase therapist numbers and optimise older patients' access to evidence-based hospital rehabilitation programmes have been strengthened by WHO's recent Rehabilitation 2030: Call to Action [1]. But as the pressure increases for health systems to provide universal coverage with access to rehabilitation it is likely that community-based rehabilitation will become more important as a cost-effective way to deliver services. Globally, community rehabilitation is also likely to become the focus for future research efforts to maximise recovery, partly because of the long trajectories of recovery (particularly of mobility) after hip fracture and partly because older people are increasingly vocal about prioritising returning home as quickly as possible.

\subsection{Conclusion}

- A rehabilitation pathway includes: (1) early and intensive mobility and self-care retraining with medical minimisation of complications and problems from comorbidities; (2) chronic care interventions (including dementia and frailty assessment and falls prevention) and (3) access to community services, including aged care support services and allied health therapies.

- Patients with cognitive impairment should not be excluded from rehabilitation following hip fracture.

- Recovery time for different functional domains varies from less than 6 months for many activities of daily living and cognitive function to over a year for walking $3 \mathrm{~m}$ without assistance.

- Rehabilitation programmes should be multidisciplinary with integration of orthogeriatric and rehabilitation services, or include professionals with multidisciplinary competencies. They should include early comprehensive geriatric assessment, surgery and mobilisation, with higher doses of mobility training and an emphasis on regaining functional independence. Programmes should also begin soon after hospital admission and continue after hospital discharge.

- In-patient rehabilitation programmes should include goal-directed mobilisation practice with balance and functional exercises.

- Structured exercise programmes should continue beyond the hospital setting for at least 12 weeks and may include progressive resistance training.

- Exercise programmes should incorporate components targeting self-efficacy to support patients to build their confidence to undertake exercise programmes post-discharge.

- Where possible a chronic disease self-management approach should be used with patients and families to promote self-efficacy and adherence to falls prevention strategies, osteoporosis treatment and exercise programmes.

- Self-efficacy, social support and caregiver responses play a role in recovery and can assist in rehabilitation in hospital and at home. Caregivers should be included in every phase of the recovery e.g. during care and discharge planning. 
- Whilst there are many barriers to providing rehabilitation services in lower to middle income countries, implementation of evidence-based recommendations should not be viewed as futile. Health care professionals at all levels should not accept cognitive and physical functioning limitations as a normal age-related pathway for older patients after hip fracture surgery.

\section{References}

1. World Health Organisation (2017) Rehabilitation 2030: a call for action. WHO, Geneva

2. Fortinsky RH, Bohannon RW, Litt MD, Tennen H, Maljanian R, Fifield J, Garcia RI, Kenyon L (2002) Rehabilitation therapy self-efficacy and functional recovery after hip fracture. Int J Rehabil Res 25(3):241-246

3. Schwarzer R, Luszczynska A, Ziegelmann JP, Scholz U, Lippke S (2008) Social-cognitive predictors of physical exercise adherence: three longitudinal studies in rehabilitation. Health Psychol 27(Suppl 1):S54-S63

4. National Institute for Health and Care Excellence (2014) Hip fracture: management. NICE Guidelines, vol CG124. National Institute for Health and Care Excellence (NICE), London

5. World Health Organisation (2011) World report on disability. World Health Organisation, Geneva

6. Crotty M, Unroe K, Cameron ID, Miller M, Ramirez G, Couzner L (2010) Rehabilitation interventions for improving physical and psychosocial functioning after hip fracture in older people. Cochrane Database Syst Rev (1):CD007624

7. Diong J, Allen N, Sherrington C (2016) Structured exercise improves mobility after hip fracture: a meta-analysis with meta-regression. Br J Sports Med 50(6):346-355

8. Handoll HH, Sherrington C, Mak JC (2011) Interventions for improving mobility after hip fracture surgery in adults. Cochrane Database Syst Rev (3):CD001704

9. Tian M, Gong X, Rath S, Wei J, Yan LL, Lamb SE, Lindley RI, Sherrington C, Willett K, Norton R (2016) Management of hip fractures in older people in Beijing: a retrospective audit and comparison with evidence-based guidelines and practice in the UK. Osteoporos Int 27(2):677-681

10. World Health Organisation (2002) Towards a common language for functioning, disability and health: ICF. The International Classification of Functioning, Disability and Health. WHO, Geneva

11. Dyer SM, Crotty M, Fairhall N, Magaziner J, Beaupre LA, Cameron ID, Sherrington C (2016) A critical review of the long-term disability outcomes following hip fracture. BMC Geriatr 16(1):158

12. Magaziner J, Hawkes W, Hebel JR, Zimmerman SI, Fox KM, Dolan M, Felsenthal G, Kenzora J (2000) Recovery from hip fracture in eight areas of function. J Gerontol A Biol Sci Med Sci 55(9):M498-M507

13. Magaziner J, Simonsick EM, Kashner TM, Hebel JR, Kenzora JE (1990) Predictors of functional recovery one year following hospital discharge for hip fracture: a prospective study. J Gerontol 45(3):M101

14. Lima CA, Sherrington C, Guaraldo A, SAD M, RDR V, JDA M, Kojima KE, Perracini M (2016) Effectiveness of a physical exercise intervention program in improving functional mobility in older adults after hip fracture in later stage rehabilitation: protocol of a randomized clinical trial (REATIVE Study). BMC Geriatr 16(1):198-198

15. Wu X, Tian M, Zhang J, Yang M, Gong X, Liu Y, Li X, Lindley RI, Anderson M, Peng K, Jagnoor J, Ji J, Wang M, Ivers R, Tian W (2019) The effect of a multidisciplinary co-management program for the older hip fracture patients in Beijing: a "pre- and post-" retrospective study. Arch Osteoporos 14(1):43

16. Beaupre LA, Cinats JG, Jones CA, Scharfenberger AV, Johnston DWC, Senthilselvan A, Saunders LD (2007) Does functional recovery in elderly hip fracture patients differ between patients admitted from long-term care and the community? J Gerontol A Biol Sci Med Sci 62(10):1127-1133 
17. Hannan E, Magaziner J, Wang J, Eastwood E (2001) Mortality and locomotion 6 months after hospitalization for hip fracture: risk factors and risk-adjusted hospital outcomes. JAMA 285(21):2736-2742

18. Seitz DP, Gill SS, Gruneir A, Austin PC, Anderson GM, Bell CM, Rochon PA (2014) Effects of dementia on postoperative outcomes of older adults with hip fractures: a population-based study. J Am Med Dir Assoc 15(5):334-341

19. Allen J, Koziak A, Buddingh S, Liang J, Buckingham J, Beaupre LA (2012) Rehabilitation in patients with dementia following hip fracture: a systematic review. Physiother Can 64(2):190-201

20. Osnes E, Lofthus K, Meyer C, Falch M, Nordsletten H, Cappelen E, Kristiansen J, Kristiansen A, Kristiansen L, Kristiansen I, Kristiansen S (2004) Consequences of hip fracture on activities of daily life and residential needs. Osteoporos Int 15(7):567-574

21. Marcantonio ER, Flacker JM, Michaels M, Resnick NM (2000) Delirium is independently associated with poor functional recovery after hip fracture. J Am Geriatr Soc 48(6):618-624

22. Goisser S, Schrader E, Singler K, Bertsch T, Gefeller O, Biber R, Bail HJ, Sieber CC, Volkert D (2015) Malnutrition according to mini nutritional assessment is associated with severe functional impairment in geriatric patients before and up to 6 months after hip fracture. J Am Med Dir Assoc 16(8):661-667

23. Goisser S, Schrader E, Singler K, Bertsch T, Gefeller O, Biber R, Bail HJ, Sieber CC, Volkert D (1940-1950) Low postoperative dietary intake is associated with worse functional course in geriatric patients up to 6 months after hip fracture. Br J Nutr 113(12):2015

24. Avenell A, Smith TO, Curtain JP, Mak JCS, Myint PK (2016) Nutritional supplementation for hip fracture aftercare in older people. Cochrane Database Syst Rev (11):CD001880

25. Neuman MD, Silber JH, Magaziner JS, Passarella MA, Mehta S, Werner RM (2014) Survival and functional outcomes after hip fracture among nursing home residents. JAMA Intern Med 174(8):1273-1280

26. Alberta Health Services. http://www.albertahealthservices.ca/assets/about/scn/ahs-scn-bjhhf-restorative-care-pathway-hcp.pdf. Accessed 1 May 2019

27. French DD, Bass E, Bradham DD, Campbell RR, Rubenstein LZ (2008) Rehospitalization after hip fracture: predictors and prognosis from a national veterans study. J Am Geriatr Soc 56(4):705-710

28. Australian and New Zealand Hip Fracture Registry (ANZHFR) Steering Group (2014) Australian and New Zealand guideline for hip fracture care: improving outcomes in hip fracture management of adults. Australian and New Zealand Hip Fracture Registry Steering Group, Sydney

29. National Clinical Guideline Centre (2011) The management of hip fracture in adults. London, National Clinical Guideline Centre

30. Mitchell SL, Stott DJ, Martin BJ, Grant SJ (2001) Randomized controlled trial of quadriceps training after proximal femoral fracture. Clin Rehabil 15(3):282-290

31. Monticone M, Ambrosini E, Brunati R, Capone A, Pagliari G, Secci C, Zatti G, Ferrante S (2018) How balance task-specific training contributes to improving physical function in older subjects undergoing rehabilitation following hip fracture: a randomized controlled trial. Clin Rehabil 32(3):340-351

32. Baroni M, Serra R, Boccardi V, Ercolani S, Zengarini E, Casucci P, Valecchi R, Rinonapoli G, Caraffa A, Mecocci P, Ruggiero C (2019) The orthogeriatric comanagement improves clinical outcomes of hip fracture in older adults. Osteoporos Int 30(4):907-916

33. Chong TW, Chan G, Feng L, Goh S, Hew A, Ng TP, Tan BY (2013) Integrated care pathway for hip fractures in a subacute rehabilitation setting. Ann Acad Med Singap 42(11):579-584

34. Fordham R, Thompson R, Holmes J, Hodkinson C (1986) A cost-benefit study of geriatric-orthopaedic management of patients with fractured neck of femur. Working papers 014. CHEDP, Centre for Health Economics, University of York, York

35. Galvard H, Samuelsson SM (1995) Orthopedic or geriatric rehabilitation of hip fracture patients: a prospective, randomized, clinically controlled study in Malmo, Sweden. Aging (Milano) 7(1):11-16 
36. Gilchrist WJ, Newman RJ, Hamblen DL, Williams BO (1988) Prospective randomised study of an orthopaedic geriatric inpatient service. BMJ 297(6656):1116-1118

37. Kennie DC, Reid J, Richardson IR, Kiamari AA, Kelt C (1988) Effectiveness of geriatric rehabilitative care after fractures of the proximal femur in elderly women: a randomised clinical trial. BMJ 297(6656):1083-1086

38. Marcantonio ER, Flacker JM, Wright RJ, Resnick NM (2001) Reducing delirium after hip fracture: a randomized trial. J Am Geriatr Soc 49(5):516-522

39. Naglie G, Tansey C, Kirkland JL, Ogilvie-Harris DJ, Detsky AS, Etchells E, Tomlinson G, O'Rourke K, Goldlist B (2002) Interdisciplinary inpatient care for elderly people with hip fracture: a randomized controlled trial. CMAJ 167(1):25-32

40. Prestmo A, Hagen G, Sletvold O, Helbostad JL, Thingstad P, Taraldsen K, Lydersen S, Halsteinli V, Saltnes T, Lamb SE, Johnsen LG, Saltvedt I (2015) Comprehensive geriatric care for patients with hip fractures: a prospective, randomised, controlled trial. Lancet 385(9978): 1623-1633

41. Sánchez Ferrín P, Mañas M, Márquez A, Dejoz M, Quintana S, González F (1999) Valoración geriátrica en ancianos con fractura proximal de fémur. Rev Esp Geriatr Gerontol 34(2):65-71

42. Stenvall M, Olofsson B, Nyberg L, Lundstrom M, Gustafson Y (2007) Improved performance in activities of daily living and mobility after a multidisciplinary postoperative rehabilitation in older people with femoral neck fracture: a randomized controlled trial with 1-year followup. J Rehabil Med 39(3):232-238

43. Uy C, Kurrle SE, Cameron ID (2008) Inpatient multidisciplinary rehabilitation after hip fracture for residents of nursing homes: a randomised trial. Australas J Ageing 27(1):43-44

44. Watne LO, Torbergsen AC, Conroy S, Engedal K, Frihagen F, Hjorthaug GA, Juliebo V, Raeder J, Saltvedt I, Skovlund E, Wyller TB (2014) The effect of a pre- and postoperative orthogeriatric service on cognitive function in patients with hip fracture: randomized controlled trial (Oslo Orthogeriatric Trial). BMC Med 12:63

45. Cameron ID, Lyle DM, Quine S (1993) Accelerated rehabilitation after proximal femoral fracture: a randomized controlled trial. Disabil Rehabil 15(1):29-34

46. Crotty M, Whitehead C, Miller M, Gray S (2003) Patient and caregiver outcomes 12 months after home-based therapy for hip fracture: a randomized controlled trial. Arch Phys Med Rehabil 84(8):1237-1239

47. Huusko TM, Karppi P, Avikainen V, Kautiainen H, Sulkava R (2002) Intensive geriatric rehabilitation of hip fracture patients: a randomized, controlled trial. Acta Orthop Scand 73(4):425-431

48. Jette AM, Harris BA, Cleary PD, Campion EW (1987) Functional recovery after hip fracture. Arch Phys Med Rehabil 68(10):735-740

49. Karlsson A, Berggren M, Gustafson Y, Olofsson B, Lindelof N, Stenvall M (2016) Effects of geriatric interdisciplinary home rehabilitation on walking ability and length of hospital stay after hip fracture: a randomized controlled trial. J Am Med Dir Assoc 17(5):464. e469-464.e415

50. Shyu YI, Liang J, Wu CC, Su JY, Cheng HS, Chou SW, Chen MC, Yang CT (2008) Interdisciplinary intervention for hip fracture in older Taiwanese: benefits last for 1 year. $\mathrm{J}$ Gerontol A Biol Sci Med Sci 63(1):92-97

51. Shyu YI, Liang J, Tseng MY, Li HJ, Wu CC, Cheng HS, Yang CT, Chou SW, Chen CY (2013) Comprehensive care improves health outcomes among elderly Taiwanese patients with hip fracture. J Gerontol A Biol Sci Med Sci 68(2):188-197

52. Singh NA, Quine S, Clemson LM, Williams EJ, Williamson DA, Stavrinos TM, Grady JN, Perry TJ, Lloyd BD, Smith EU, Singh MA (2012) Effects of high-intensity progressive resistance training and targeted multidisciplinary treatment of frailty on mortality and nursing home admissions after hip fracture: a randomized controlled trial. J Am Med Dir Assoc 13(1):24-30

53. Swanson CE, Day GA, Yelland CE, Broome JR, Massey L, Richardson HR, Dimitri K, Marsh A (1998) The management of elderly patients with femoral fractures. A randomised controlled trial of early intervention versus standard care. Med J Aust 169(10):515-518 
54. Vidan M, Serra JA, Moreno C, Riquelme G, Ortiz J (2005) Efficacy of a comprehensive geriatric intervention in older patients hospitalized for hip fracture: a randomized, controlled trial. J Am Geriatr Soc 53(9):1476-1482

55. Ziden L, Frandin K, Kreuter M (2008) Home rehabilitation after hip fracture. A randomized controlled study on balance confidence, physical function and everyday activities. Clin Rehabil 22(12):1019-1033

56. Crotty M, Killington M, Liu E, Cameron ID, Kurrle S, Kaambwa B, Davies O, Miller M, Chehade M, Ratcliffe J (2019) Should we provide outreach rehabilitation to very old people living in Nursing Care Facilities after a hip fracture? A randomised controlled trial. Age Ageing 48(3):373-380

57. Ryan T, Enderby P, Rigby AS (2006) A randomized controlled trial to evaluate intensity of community-based rehabilitation provision following stroke or hip fracture in old age. Clin Rehabil 20(2):123-131

58. Kimmel LA, Liew SM, Sayer JM, Holland AE (2016) HIP4Hips (High Intensity Physiotherapy for HIP fractures in the acute hospital setting): a randomised controlled trial. Med J Aust 205(2):73-78

59. Kronborg L, Bandholm T, Palm H, Kehlet H, Kristensen MT (2017) Effectiveness of acute in-hospital physiotherapy with knee-extension strength training in reducing strength deficits in patients with a hip fracture: a randomised controlled trial. PLoS One 12(6):e0179867

60. Moseley AM, Sherrington C, Lord SR, Barraclough E, St George RJ, Cameron ID (2009) Mobility training after hip fracture: a randomised controlled trial. Age Ageing 38(1):74-80

61. Ohoka T, Urabe Y, Shirakawa T (2015) Therapeutic exercises for proximal femoral fracture of super-aged patients: effect of walking assistance using Body Weight-Supported Treadmill Training (BWSTT). Physiotherapy 101:e1124-e1125

62. Resnick B, Magaziner J, Orwig D, Yu-Yahiro J, Hawkes W, Shardell M, Hebel JR, Zimmerman S, Golden J, Werner M (2007) Testing the effectiveness of the exercise plus program in older women post-hip fracture. Ann Behav Med 34(1):67-76

63. Sherrington C, Lord SR, Herbert RD (2003) A randomised trial of weight-bearing versus non-weight-bearing exercise for improving physical ability in inpatients after hip fracture. Aust J Physiother 49(1):15-22

64. Van Ooijen MLW, Roerdink M, Trekop M, Janssen TWJ, Beek PJ (2016) The efficacy of treadmill training with and without projected visual context for improving walking ability and reducing fall incidence and fear of falling in older adults with fall-related hip fracture: a randomized controlled trial. BMC Geriatr 16(1):215

65. Binder EF, Brown M, Sinacore DR, Steger-May K, Yarasheski KE, Schechtman KB (2004) Effects of extended outpatient rehabilitation after hip fracture: a randomized controlled trial. JAMA 292(7):837-846

66. Hauer K, Specht N, Schuler M, Bärtsch P, Oster P (2002) Intensive physical training in geriatric patients after severe falls and hip surgery. Age Ageing 31(1):49-57

67. Langford D, Fleig L, Brown K, Cho N, Frost M, Ledoyen M, Lehn J, Panagiotopoulos K, Sharpe N, Ashe MC (2015) Back to the future-feasibility of recruitment and retention to patient education and telephone follow-up after hip fracture: a pilot randomized controlled trial. Patient Prefer Adherence 2015:1343-1351

68. Latham NK, Harris BA, Bean JF, Heeren T, Goodyear C, Zawacki S, Heislein DM, Mustafa J, Pardasaney P, Giorgetti M, Holt N, Goehring L, Jette AM (2014) Effect of a home-based exercise program on functional recovery following rehabilitation after hip fracture: a randomized clinical trial. JAMA 311(7):700-708

69. Magaziner J, Mangione KK, Orwig D, Baumgarten M, Magder L, Terrin M, Fortinsky RH, Gruber-Baldini AL, Beamer BA, Tosteson ANA, Kenny AM, Shardell M, Binder EF, Koval K, Resnick B, Miller R, Forman S, McBride R, Craik RL (2019) Effect of a multicomponent home-based physical therapy intervention on ambulation after hip fracture in older adults: the CAP randomized clinical trial. JAMA 322(10):946-956

70. Mangione KK, Craik RL, Tomlinson SS, Palombaro KM (2005) Can elderly patients who have had a hip fracture perform moderate- to high-intensity exercise at home? Phys Ther 85(8):727-739 
71. Mangione KK, Craik RL, Palombaro KM, Tomlinson SS, Hofmann MT (2010) Home-based leg-strengthening exercise improves function 1 year after hip fracture: a randomized controlled study. J Am Geriatr Soc 58(10):1911-1917

72. Orwig D, Hochberg M, Yu-Yahiro J, Resnick B, Hawkes W, Shardell M, Hebel J, Colvin P, Miller R, Golden J, Zimmerman S, Magaziner J (2011) Delivery and outcomes of a yearlong home exercise program after hip fracture: a randomized controlled trial. Arch Intern Med 171(4):323

73. Salpakoski A, Törmäkangas T, Edgren J, Kallinen M, Sihvonen SE, Pesola M, Vanhatalo J, Arkela M, Rantanen T, Sipilä S (2014) Effects of a multicomponent home-based physical rehabilitation program on mobility recovery after hip fracture: a randomized controlled trial. J Am Med Dir Assoc 15(5):361-368

74. Sherrington C, Lord SR (1997) Home exercise to improve strength and walking velocity after hip fracture: a randomized controlled trial. Arch Phys Med Rehabil 78(2):208-212

75. Sherrington C, Lord SR, Herbert RD (2004) A randomized controlled trial of weight-bearing versus non-weight-bearing exercise for improving physical ability after usual care for hip fracture. Arch Phys Med Rehabil 85(5):710-716

76. Stasi S, Papathanasiou G, Chronopoulos E, Dontas IA, Baltopoulos IP, Papaioannou NA (2019) The effect of intensive abductor strengthening on postoperative muscle efficiency and functional ability of hip-fractured patients: a randomized controlled trial. Indian J Orthop 53(3):407-419

77. Sylliaas H, Brovold T, Wyller TB, Bergland A (2011) Progressive strength training in older patients after hip fracture: a randomised controlled trial. Age Ageing 40(2):221-227

78. Sylliaas H, Brovold T, Wyller TB, Bergland A (2012) Prolonged strength training in older patients after hip fracture: a randomised controlled trial. Age Ageing 41(2):206-212

79. Tsauo JY, Leu WS, Chen YT, Yang RS (2005) Effects on function and quality of life of postoperative home-based physical therapy for patients with hip fracture. Arch Phys Med Rehabil 86(10):1953-1957

80. Williams NH, Roberts JL, Din NU, Totton N, Charles JM, Hawkes CA, Morrison V, Hoare Z, Williams M, Pritchard AW, Alexander S, Lemmey A, Woods RT, Sackley C, Logan P, Edwards RT, Wilkinson C (2016) Fracture in the Elderly Multidisciplinary Rehabilitation (FEMuR): a phase II randomised feasibility study of a multidisciplinary rehabilitation package following hip fracture. BMJ Open 6(10):e012422-e012422

81. Shyu YI, Liang J, Wu CC, Su JY, Cheng HS, Chou SW, Chen MC, Yang CT, Tseng MY (2010) Two-year effects of interdisciplinary intervention for hip fracture in older Taiwanese. J Am Geriatr Soc 58(6):1081-1089

82. Rimmer JH, Henley KY (2013) Building the crossroad between inpatient/outpatient rehabilitation and lifelong community-based fitness for people with neurologic disability. J Neurol Phys Ther 37(2):72-77

83. Chang FH, Latham NK, Ni P, Jette AM (2015) Does self-efficacy mediate functional change in older adults participating in an exercise program after hip fracture? A randomized controlled trial. Arch Phys Med Rehabil 96(6):1014-1020

84. Bean JF, Herman S, Kiely DK, Frey IC, Leveille SG, Fielding RA, Frontera WR (2004) Increased Velocity Exercise Specific to Task (InVEST) training: a pilot study exploring effects on leg power, balance, and mobility in community-dwelling older women. J Am Geriatr Soc 52(5):799-804

85. Seitz DP, Adunuri N, Gill SS, Rochon PA (2011) Prevalence of dementia and cognitive impairment among older adults with hip fractures. J Am Med Dir Assoc 12(8):556-564

86. Royal College of Physicians (2018) National hip fracture database (NHFD). Royal College of Physicians, London

87. National Clinical Guideline Centre (2017) The management of hip fracture in adults. London, National Clinical Guideline Centre

88. Freter S, Koller K, Dunbar M, MacKnight C, Rockwood K (2017) Translating delirium prevention strategies for elderly adults with hip fracture into routine clinical care: a pragmatic clinical trial. J Am Geriatr Soc 65(3):567-573

89. Stenvall M, Berggren M, Lundstrom M, Gustafson Y, Olofsson B (2012) A multidisciplinary intervention program improved the outcome after hip fracture for people with 
dementia — subgroup analyses of a randomized controlled trial. Arch Gerontol Geriatr 54(3): e284-e289

90. Huusko TM, Karppi P, Avikainen V, Kautiainen H, Sulkava R (2000) Randomised, clinically controlled trial of intensive geriatric rehabilitation in patients with hip fracture: subgroup analysis of patients with dementia. BMJ 321(7269):1107-1111

91. Shyu YI, Tsai WC, Chen MC, Liang J, Cheng HS, Wu CC, Su JY, Chou SW (2012) Twoyear effects of an interdisciplinary intervention on recovery following hip fracture in older Taiwanese with cognitive impairment. Int J Geriatr Psychiatry 27(5):529-538

92. Bischoff-Ferrari HA, Dawson-Hughes B, Platz A, Orav EJ, Stähelin HB, Willett WC, Can U, Egli A, Mueller NJ, Looser S, Bretscher B, Minder E, Vergopoulos A, Theiler R (2010) Effect of high-dosage cholecalciferol and extended physiotherapy on complications after hip fracture: a randomized controlled trial. Arch Intern Med 170(9):813-820

93. Liu H, Yang C, Tseng M, Chen C, Wu C, Cheng H, Lin Y, Shyu Y (2018) Trajectories in postoperative recovery of elderly hip-fracture patients at risk for depression: a follow-up study. Rehabil Psychol 63(3):438-446

94. Cristancho P, Lenze EJ, Avidan MS, Rawson KS (2016) Trajectories of depressive symptoms after hip fracture. Psychol Med 46(7):1413-1425

95. Moraes SA, Furlanetto EC, Ricci NA, Perracini MR (2019) Sedentary behavior: barriers and facilitators among older adults after hip fracture surgery. A qualitative study. Braz J Phys Ther. https://www.sciencedirect.com/science/article/abs/pii/S1413355518310232?via\%3Dihub. Accessed 10 July 2019

96. Bower ES, Wetherell JL, Petkus AJ, Rawson KS, Lenze EJ (2016) Fear of falling after hip fracture: prevalence, course, and relationship with one-year functional recovery. Am J Geriatr Psychiatry 24(12):1228-1236

97. Visschedijk J, van Balen R, Hertogh C, Achterberg W (2013) Fear of falling in patients with hip fractures: prevalence and related psychological factors. J Am Med Dir Assoc 14(3):218-220

98. Lim KK, Matchar DB, Chong JL, Yeo W, Howe TS, Koh JSB (2019) Pre-discharge prognostic factors of physical function among older adults with hip fracture surgery: a systematic review. Osteoporos Int 30(5):929-938

99. Shyu YI, Chen MC, Wu CC, Cheng HS (2010) Family caregivers' needs predict functional recovery of older care recipients after hip fracture. J Adv Nurs 66(11):2450-2459

100. Salkeld G, Cameron ID, Cumming RG, Easter S, Seymour J, Kurrle SE, Quine S (2000) Quality of life related to fear of falling and hip fracture in older women: a time trade off study. BMJ 320(7231):341-346

101. Schwartz CE, Bode R, Repucci N, Becker J, Sprangers MA, Fayers PM (2006) The clinical significance of adaptation to changing health: a meta-analysis of response shift. Qual Life Res 15(9):1533-1550

102. Pol M, Peek S, van Nes F, van Hartingsveldt M, Buurman B, Krose B (2019) Everyday life after a hip fracture: what community-living older adults perceive as most beneficial for their recovery. Age Ageing 48(3):440-447

103. Langford D, Edwards N, Gray SM, Fleig L, Ashe MC (2018) "Life goes on" everyday tasks, coping self-efficacy, and independence: exploring older adults' recovery from hip fracture. Qual Health Res 28(8):1255-1266

104. Yu F, Xia W (2019) The epidemiology of osteoporosis, associated fragility fractures, and management gap in China. Arch Osteoporos 14(1):32

105. Rath S, Yadav L, Tewari A, Chantler T, Woodward M, Kotwal P, Jain A, Dey A, Garg B, Malhotra R, Goel A, Farooque K, Sharma V, Webster P, Norton R (2017) Management of older adults with hip fractures in India: a mixed methods study of current practice, barriers and facilitators, with recommendations to improve care pathways. Arch Osteoporos 12(1):55

106. Riera-Espinoza G (2009) Epidemiology of osteoporosis in Latin America 2008. Salud Publica Mex 51(Suppl 1):S52-S55

107. Zerbini CA, Szejnfeld VL, Abergaria BH, McCloskey EV, Johansson H, Kanis JA (2015) Incidence of hip fracture in Brazil and the development of a FRAX model. Arch Osteoporos 10:224 
108. Dhanwal DK, Dennison EM, Harvey NC, Cooper C (2011) Epidemiology of hip fracture: worldwide geographic variation. Indian J Orthop 45(1):15-22

109. Dash SK, Panigrahi R, Palo N, Priyadarshi A, Biswal M (2015) Fragility hip fractures in elderly patients in Bhubaneswar, India (2012-2014): a prospective multicenter study of 1031 elderly patients. Geriatr Orthop Surg Rehabil 6(1):11-15

110. González ID, Becerra MC, González J, Campos AT, Barbosa-Santibáñez J, Alvarado R (2016) Fracturas de cadera: satisfacción posquirúrgica al año en adultos mayores atendidos en Méderi-Hospital Universitario Mayor, Bogotá, D.C. Rev Cienc Salud 14(3):409-422

111. Arliani GG, da Costa AD, Linhares GK, Balbachevsky D, Fernandes HJ, Dos Reis FB (2011) Correlation between time until surgical treatment and mortality among elderly patients with fractures at the proximal end of the femur. Rev Bras Ortop 46(2):189-194

112. Dinamarca-Montecinos JL, Amestica-Lazcano G, Rubio-Herrera R, Carrasco-Buvinic A, Vasquez A (2015) [Hip fracture. Experience in 647 Chilean patients aged 60 years or more]. Rev Med Chil 143(12):1552-1559

113. World Health Organisation. https://www.who.int/news-room/fact-sheets/detail/rehabilitation. Accessed 22 Aug 2019

114. Lin PC, Hung SH, Liao MH, Sheen SY, Jong SY (2006) Care needs and level of care difficulty related to hip fractures in geriatric populations during the post-discharge transition period. J Nurs Res 14(4):251-260

115. Rocha SA, Avila MA, Bocchi SC (2016) The influence of informal caregivers on the rehabilitation of the elderly in the postoperative period of proximal femoral fracture. Rev Gaucha Enferm 37(1):e51069

116. Vidal EI, Coeli CM, Pinheiro RS, Camargo KR (2006) Mortality within 1 year after hip fracture surgical repair in the elderly according to postoperative period: a probabilistic record linkage study in Brazil. Osteoporos Int 17(10):1569-1576

117. Chen Y-T, Peng L-N, Liu C-L, Chen L-K (2011) Orthogeriatrics in Taiwan: overview and experiences. J Clin Gerontol Geriatr 2:66e70

118. Lima CA, Sherrington C, Guaraldo A, Moraes SA, Varanda RD, Melo JA, Kojima KE, Perracini M (2016) Effectiveness of a physical exercise intervention program in improving functional mobility in older adults after hip fracture in later stage rehabilitation: protocol of a randomized clinical trial (REATIVE Study). BMC Geriatr 16(1):198

119. Furlan AD, Irvin E, Munhall C, Giraldo-Prieto M, Fullerton L, McMaster R, Danak S, Costante A, Pitzul K, Bhide RP, Marchenko S, Mahood Q, David JA, Flannery JF, Bayley M (2018) Rehabilitation service models for people with physical and/or mental disability living in low- and middle-income countries: a systematic review. J Rehabil Med 50(6):487-498

Open Access This book is licensed under the terms of the Creative Commons AttributionNonCommercial-NoDerivatives 4.0 International License (http://creativecommons.org/licenses/ by-nc-nd/4.0/), which permits any noncommercial use, sharing, distribution and reproduction in any medium or format, as long as you give appropriate credit to the original author(s) and the source, provide a link to the Creative Commons license and indicate if you modified the licensed material. You do not have permission under this license to share adapted material derived from this book or parts of it.

The images or other third party material in this book are included in the book's Creative Commons license, unless indicated otherwise in a credit line to the material. If material is not included in the book's Creative Commons license and your intended use is not permitted by statutory regulation or exceeds the permitted use, you will need to obtain permission directly from the copyright holder.

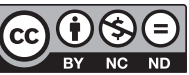

